\title{
Modeling Task Switching Without Switching Tasks: A Short-Term Priming Account of Explicitly Cued Performance
}

\author{
Darryl W. Schneider and Gordon D. Logan \\ Vanderbilt University
}

\begin{abstract}
Switch costs in task switching are commonly attributed to an executive control process of task-set reconfiguration, particularly in studies involving the explicit task-cuing procedure. The authors propose an alternative account of explicitly cued performance that is based on 2 mechanisms: priming of cue encoding from residual activation of cues in short-term memory and compound cue retrieval of responses from long-term memory. Their short-term priming account explains the repeated cue encoding benefit, switch cost, reduction in switch cost with preparation time, and other effects. The authors develop a mathematical model of their priming account and fit it to data from 3 experiments, demonstrating that a set of basic psychological processes can produce several effects-including putative switch costswithout switching tasks.
\end{abstract}

Keywords: task switching, switch cost, priming, compound cue retrieval

A remarkable property of the human cognitive system is its flexibility. As human beings, we can adapt to changing circumstances in a fraction of a second, often using external cues as indicators of change. However, interpreting these cues in the context of the other stimuli available to our senses is not always straightforward, and occasionally it seems as if a modicum of control is required to guide our actions. We are then confronted with a fundamental issue in cognitive psychology: how the mind controls itself. Many complex cognitive processes have been attributed to mechanisms of executive control, such as selecting and modifying task goals, devising strategies to achieve these goals, programming and coordinating the subordinate processes needed to implement the strategies, and monitoring and troubleshooting performance (Logan, 1985, 2003; Monsell, 1996; Norman \& Shallice, 1986). Many situations are thought to engage executive control, with recent research focusing on task switching.

In this article, we propose a short-term priming account of explicitly cued task-switching performance that does not require an executive control mechanism to switch tasks. We develop a priming model based on our short-term priming account that is written in the language of Logan's (2002) instance theory of attention and memory, which integrates Bundesen's (1990) theory of visual attention, Logan and Gordon's (2001) theory of executive control, Nosofsky's (1986) generalized context model of categorization, Logan's (1988) instance theory of automaticity, and

Additional materials are on the Web at http://dx.doi.org/10.1037/00963445.134.3.343.supp

This research was supported by National Science Foundation Grant BCS 0133202 to Gordon D. Logan. We thank Andrew Tomarken for statistical advice and Stephen Monsell and Sander Nieuwenhuis for comments on earlier versions of this article.

Correspondence concerning this article should be addressed to Darryl W. Schneider or Gordon D. Logan, Department of Psychology, Vanderbilt University, 111 21st Avenue South, Nashville, TN 37203. E-mail: darryl.schneider@vanderbilt.edu or gordon.logan@vanderbilt.edu
Nosofsky and Palmeri's (1997) exemplar-based random walk model in a common mathematical framework. These theories account for numerous phenomena in cognitive psychology, and our priming model extends their coverage to the task-switching domain with the modeling of cue encoding and response selection processes. We develop the modeling of cue encoding processes (Arrington \& Logan, 2004; Logan \& Bundesen, 2003, 2004) in greater detail by linking them to repetition and associative priming effects in short-term memory (STM). We integrate mechanisms from models of categorization and memory retrieval (Gillund \& Shiffrin, 1984; Hintzman, 1986; Luce, 1959, 1963; Nosofsky, 1986; Raaijmakers \& Shiffrin, 1981; Shepard, 1957) with a random walk process (Nosofsky \& Palmeri, 1997; Ratcliff, 1978) to enable response selection by compound cue retrieval of response categories from long-term memory (Dosher \& Rosedale, 1989, Ratcliff \& McKoon, 1988). Modeling cue encoding and response selection processes within a formal framework shared by a family of models in the attention and memory literature (Logan, 2002, 2004) allows us to maintain connectivity with past research and build a stronger foundation for future research.

Our exposition begins with an overview of task switching and the explicit task-cuing procedure, outlining key findings and theoretical interpretations. We then introduce our priming account and describe a mathematical model that instantiates its assumptions and mechanisms. We demonstrate that our priming model can account for the critical effects from three experiments without invoking executive control processes to switch tasks. We conclude by discussing applications of the priming model, its limitations and possible extensions, and implications concerning executive control, task sets, and strategies for theory development.

\section{Task Switching}

\section{Overview}

Task switching is a common paradigm for studying executive control in cognitive psychology (e.g., Allport, Styles, \& Hsieh, 
1994; Meiran, 1996; Rogers \& Monsell, 1995), cognitive neuroscience (e.g., Brass \& von Cramon, 2004; Sohn, Ursu, Anderson, Stenger, \& Carter, 2000; Stoet \& Snyder, 2004), clinical science (e.g., Meiran, Levine, Meiran, \& Henik, 2000; Woodward, Bub, \& Hunter, 2002), and aging (e.g., Kramer, Hahn, \& Gopher, 1999; Mayr, 2001; Salthouse, Fristoe, McGuthry, \& Hambrick, 1998). Task switching involves performing different tasks in alternation, which is compared with performing the same task repeatedly. Performance is measured in terms of response time (RT) and accuracy, with the robust finding that responses are slower and less accurate when one is alternating tasks than when one is repeating tasks (e.g., Allport et al., 1994; Jersild, 1927; Meiran, 1996; Rogers \& Monsell, 1995; Rubinstein, Meyer, \& Evans, 2001; Spector \& Biederman, 1976; for a review, see Monsell, 2003). The difference in RT between task alternations and task repetitions is interpreted as a switch cost related to the implementation of a task set-a specific configuration of the cognitive system-in STM (Monsell, 2003). From this perspective, the task set must change for task alternations, but not for task repetitions.

The predominant goal among researchers who study task switching is to explain switch costs. In the past 10 years, two primary accounts have emerged: task-set reconfiguration and taskset priming. Proponents of task-set reconfiguration argue that switch costs arise from an endogenous, time-consuming executive control process that reconfigures the cognitive system to implement the relevant task set for task alternations (De Jong, 2000; Meiran, 1996; Monsell, 1996; Rogers \& Monsell, 1995; Rubinstein et al., 2001). An implicit assumption of task-set reconfiguration is that once a task set is implemented, it remains in the same state of activation until it has to be changed. Task repetitions benefit from this prepared state because the relevant task set is in place and no reconfiguration is necessary, resulting in faster performance relative to task alternations. In this context, switch costs could be construed as repetition benefits (see Ruthruff, Remington, \& Johnston, 2001), although the former term is often used to emphasize the perceived role of executive control processes.

Proponents of task-set priming argue that switch costs arise in part from proactive interference and associative priming of task sets and stimulus-response bindings across trials (e.g., Allport et al., 1994; Allport \& Wylie, 1999, 2000; Waszak, Hommel, \& Allport, 2003, 2004; Wylie \& Allport, 2000). Task-set priming is based on the idea that associations learned between stimuli and responses or tasks are retrieved and affect performance on any given trial. Collections of stimulus-response or stimulus-task bindings could be viewed as task sets, with residual activation of these task sets interfering with the implementation of the relevant task set for task alternations, resulting in slower responses (Allport et al., 1994). Item-specific stimulus-response bindings also could contribute to switch costs, with negative priming of responses from retrieval of competing responses (Allport \& Wylie, 2000; Koch \& Allport, in press; Waszak et al., 2003; Wylie \& Allport, 2000).

Task-set priming theorists do not exclude executive control from task switching, and there are authors who have argued that priming and executive control processes both contribute to performance (e.g., Goschke, 2000; Meiran, 2000a, 2000b; Meiran, Chorev, \& Sapir, 2000; Sohn \& Anderson, 2001, 2003). Some researchers have characterized stimulus-task bindings as "control states" (Allport \& Wylie, 1999, p. 293), arguing that priming comes from an interaction between top-down and bottom-up fac- tors. In models of task-set priming, switch costs are generated from an interaction between task-set priming and executive control input (Gilbert \& Shallice, 2002; Yeung \& Monsell, 2003). The main difference between these models and task-set reconfiguration is that executive control input is required on all trials; there is not an executive control process that occurs only for task alternations. If executive control is involved with both task alternations and task repetitions, then switch cost is not a valid measure of the duration of an executive control process.

\section{The Explicit Task-Cuing Procedure}

The focus of the present article is on task-set reconfiguration, questioning whether the difference between task alternations and task repetitions has to be interpreted as a switch cost reflecting the duration of an executive control process. We examined this issue with a method that has been frequently interpreted as providing measures of task-set reconfiguration: the explicit task-cuing procedure (Shaffer, 1965; Sudevan \& Taylor, 1987). A cue indicating the task to be performed is presented prior to the target stimulus on each trial. Subjects must respond to the target based on the task signaled by the cue; the target alone is usually ambiguous. For example, if the tasks are to classify digits as odd or even and higher or lower than 5, the target 3 could be classified as odd or low. A task cue (e.g., ODD-EVEN) accompanying the target allows an unambiguous response to be selected.

An advantage of the explicit task-cuing procedure is the ability to manipulate the delay between the onsets of the cue and targetthe stimulus onset asynchrony (SOA). Many researchers have manipulated SOA to study preparatory processes in task switching (e.g., Goschke, 2000; Koch, 2003; Logan \& Bundesen, 2003 , 2004; Mayr \& Kliegl, 2000, 2003; Meiran, 1996, 2000a; Meiran, Chorev, \& Sapir, 2000; Monsell, Sumner, \& Waters, 2003). The cue allows for task-set reconfiguration prior to target onset. If SOA is construed as time available for this advance preparation, then increasing SOA should increase the chances of completing task-set reconfiguration, reducing switch costs. There is evidence that switch cost decreases with increasing SOA (but see Altmann, 2004), suggesting that cue-initiated task-set reconfiguration is possible.

Much of the support for task-set reconfiguration is contingent on how the explicit task-cuing procedure is interpreted. Until recently, many researchers did not consider a critical aspect of the procedure: Cue repetitions were confounded with task repetitions. With a single cue assigned to each task, a task repetition always involved a cue repetition, whereas a task alternation always involved a cue alternation. The switch costs observed in previous studies could be reinterpreted as benefits of repeated cue encoding-a process that does not require executive control (Logan \& Bundesen, 2003). If switch costs are nothing more than differences in cue encoding, then the task-set reconfiguration view is weakened and the utility of the explicit task-cuing procedure for studying executive control is placed in jeopardy (Logan, 2003).

Logan and Bundesen (2003) formally modeled task-set reconfiguration and cue encoding processes to account for data from experiments using the explicit task-cuing procedure with masked cues (Experiments 1 and 2), multiple cues per task (Experiments 3 and 4), and varying intertrial intervals (Experiment 5). Three models were developed: Model 1, which assumed there was task- 
set reconfiguration only; Model 2, which assumed there was a repeated cue encoding benefit but no task-set reconfiguration; and Model $2+1$, which assumed that both processes influenced performance. Across the five experiments, Model 1 provided the poorest fits to the data, whereas Models 2 and $2+1$ fared much better. Logan and Bundesen considered Model 2 to provide the best account of the data because Model $2+1$ violated certain assumptions and produced what they deemed to be unreasonable parameter estimates in some cases. Logan and Bundesen argued that switch costs in the explicit task-cuing procedure do not reflect executive control.

\section{Fractionating "Switch Cost” With Multiple Cues per Task}

An important feature of the study by Logan and Bundesen (2003) is their use of two cues per task in Experiments 3 and 4. This design allows three types of transitions: cue repetitions (both cue and task repeat), task repetitions (task repeats but cue changes), and task alternations (both cue and task change). Previous studies using a single cue per task only allowed cue repetitions and task alternations. With multiple cues per task, the difference between cue repetitions and task repetitions can be interpreted as a repeated cue encoding benefit, whereas the difference between task repetitions and task alternations can be interpreted as a switch cost that is uncontaminated by cue repetitions. Logan and Bundesen (2003) found substantial differences between cue repetitions and task repetitions, but only small differences between task repetitions and task alternations. They argued that performance was based on a compound stimulus strategy, whereby subjects retrieved a unique response from memory based on the combination of the cue and the target. Such a strategy does not require executive control but would benefit from repeated cue encoding (also see Arrington \& Logan, 2004).

Mayr and Kliegl (2003) also conducted experiments using the explicit task-cuing procedure and multiple cues per task and obtained different results than Logan and Bundesen (2003). Mayr and Kliegl (2003) found a substantial difference not only between cue repetitions and task repetitions but also between task repetitions and task alternations. They proposed a two-stage model to account for their data: cue-initiated retrieval of task rules from long-term memory (retrieval stage) and application of the rules to the target to generate a response (application stage). Mayr and Kliegl (2003) argued that the retrieval stage produced the difference between cue repetitions and task repetitions due to priming of the retrieval process for cue repetitions. They argued that the application stage produced the difference between task repetitions and task alternations, whereby the automatic application of task rules to the target takes longer when switching tasks. Mayr and Kliegl's (2003) and Logan and Bundesen's (2003) studies indicated that much of the "switch cost" observed in previous research was due to cue-related processing, but they differed in their interpretations and data concerning switch costs uncontaminated by cue repetition.

Logan and Bundesen (2004) addressed the discrepancy between the two earlier studies by manipulating the type of cues associated with the tasks. Mayr and Kliegl (2003) used arbitrary letter cues, whereas Logan and Bundesen (2003) used meaningful word cues. Previous research has demonstrated that the types of cues associated with tasks influence switch costs; meaningful word cues produce smaller switch costs than spatial or shape cues (Arbuth- nott \& Woodward, 2002; also see Miyake, Emerson, Padilla, \& Ahn, 2004). Logan and Bundesen (2004) argued that the abstract nature of the letter cues used by Mayr and Kliegl (2003) may have led subjects to invoke a mediating process involving access to task names in memory. The task name would then be combined with the target for retrieval of a response from memory.

To test this hypothesis, Logan and Bundesen (2004) conducted two experiments in which tasks were cued by arbitrary letters or meaningful words in separate blocks. They found little difference between task repetitions and task alternations, except when letter cues were used in the first block. These results and formal modeling indicating that Model 2 of Logan and Bundesen (2003) provided a good account of much of the data supported the idea that a compound stimulus strategy was being used, but that it required access to a mediator in the initial stages of performance. Further evidence in support of the compound stimulus strategy comes from a recent study by Arrington and Logan (2004), who investigated whether the strategy was episodic or semantic by manipulating the number of unique targets viewed by different groups of subjects. The differences among transitions were very similar across groups, leading Arrington and Logan to argue that the compound stimulus strategy was based on the cue and the target retrieving representations from semantic memory, at least initially.

Research with multiple cues per task seems to indicate that the explicit task-cuing procedure rarely involves executive control and can be explained by a compound stimulus strategy. However, the mechanisms underlying the compound stimulus strategy have not been defined, and it is unclear how the strategy can arise solely from bottom-up input. For example, in Model 2 of Logan and Bundesen (2003), differences in cue encoding account for the differences among transitions: When a cue repeats, cue encoding is faster than when a cue changes. What is the basis for differences in cue encoding? Logan and Bundesen (2003) suggested an answer to this question that we examine and extend in the present study. Moreover, statistically significant differences between task alternations and task repetitions have been observed (Arrington \& Logan, 2004; Brass \& von Cramon, 2004; Logan \& Bundesen, 2003, 2004; Mayr \& Kliegl, 2003), but Model 2 never predicts such differences. How are these differences-which are suggestive of switch costs-possible? Finally, it is important to understand how the compound stimulus strategy can produce a unique response; the underlying mechanism has yet to be modeled. How can a response be selected using the cue and the target in combination? The priming account we present in the next section provides answers to all of these questions.

\section{A Short-Term Priming Account of Explicitly Cued Performance}

We developed a short-term priming account of explicitly cued performance in task-switching situations that explains differences among transitions without invoking an executive control mechanism to switch tasks (also see Altmann, 2003, 2004). We focused on the explicit task-cuing procedure for several reasons. First, it is a common method for studying task switching (e.g., Arbuthnott \& Woodward, 2002; Arrington, Altmann, \& Carr, 2003; Arrington \& Logan, 2004; Goschke, 2000; Koch, 2003; Logan \& Bundesen, 2003, 2004; Mayr \& Kliegl, 2000, 2003; Meiran, 1996, 2000a, 
2000b; Meiran, Chorev, \& Sapir, 2000). Second, we wanted to account for situations involving unpredictable, explicitly cued transitions. Task-set priming accounts address experiments in which transitions occur predictably (in blocks, cycles, or sequenced runs) or single-task performance is studied following performance of competing tasks (e.g., Allport et al., 1994; Allport \& Wylie, 1999, 2000; Altmann, 2002; Koch \& Allport, in press; Waszak et al., 2003, 2004; Yeung \& Monsell, 2003). Moreover, computational models of task-set priming deal with situations without explicit cues (Gilbert \& Shallice, 2002; Yeung \& Monsell, 2003). Third, the explicit task-cuing procedure provides some of the strongest support for task-set reconfiguration; the reduction in switch cost with SOA suggests that task-set reconfiguration can begin prior to target onset. We demonstrate that bottom-up processes can produce the reduction in switch cost, indicating that this effect cannot be attributed unequivocally to an executive control process.

\section{Overview of the Experiments}

Before we describe our priming account, it is helpful to give an overview of the experiments that we used to assess it. We conducted three experiments using the explicit task-cuing procedure. The tasks were to classify single-digit targets as odd or even and higher or lower than 5. Each task was associated with multiple cues, permitting cue repetitions, task repetitions, and task alternations. The main difference between the present study and the studies by Logan and Bundesen $(2003,2004)$ and Mayr and Kliegl (2003) is the selection of cues and their associations to the targets and response categories. We chose cues that were strongly associated to permit a stringent assessment of our priming account: the specific response categories associated with the tasks (i.e., $O D D$ and EVEN were cues for the odd-even task and HIGH and LOW were cues for the high-low task). Two types of cue-target congruency emerge with these cues: The cue and target can be associated with the same response category (congruent; e.g., $O D D-3$ ) or different response categories (incongruent; e.g., $O D D-$ 4 ). The differences among transitions and the effects of cue-target congruency are closely linked in our priming account as described below.

\section{Basic Mechanisms and Assumptions}

Our priming account is based on two mechanisms: priming of cue encoding from residual activation of cues in STM and compound cue retrieval of response categories from long-term memory. Priming of cue encoding accounts for differences among transitions, and compound cue retrieval accounts for differences in cue-target congruency.

Priming of cue encoding. Before a cue can be used to retrieve responses from long-term memory, it must be encoded into STM. Following Logan and Bundesen (2003), cue encoding is construed as a race between comparison processes that match the perceptual representation of the presented cue to its transient representation in STM and to its permanent representation in long-term memory. Cue encoding time is determined by whichever comparison process finishes first. We assume the rate of comparison to long-term memory is constant for all transitions, but the rate of comparison to STM depends on the transition. Consequently, differences be- tween transitions are due to differences in cue encoding time, which result from different rates of comparison to STM as described later.

Once a cue is encoded, its perceptual representation serves as a retrieval cue for response categories in long-term memory. For clarity, we denote cues in uppercase italic letters (e.g., $O D D$ ) and response categories in lowercase italic letters (e.g., odd). Cues are associated with response categories with strengths that depend on the associations between response categories. We assume that odd is associated with even (and vice versa) and high is associated with low (and vice versa), but each pair of response categories is only weakly associated with the other pair. These assumptions are supported by free association norms (Nelson, McEvoy, \& Schreiber, 1999). ${ }^{1}$ The associations between response categories enable a cue to be associated with multiple response categories with varying strengths. For example, $O D D$ is strongly associated with $o d d$, partially associated with even, and unassociated with high and low. Strongly and partially associated response categories will be retrieved into STM, so $O D D$ will only retrieve odd and even, but with more strength for $o d d$ due to a stronger association. Unassociated response categories such as high and low would not be retrieved with any strength beyond some baseline level.

When a response category that is only partially associated with the presented cue is retrieved, we assume that the perceptual representations of cues strongly associated with that response category become partially activated in STM. For example, if the presented cue is $O D D$, odd and even will be retrieved, and we assume that EVEN becomes partially activated in STM due to its strong association with even. Partial activation of cues occurs indirectly through retrieval of associated response categories because it is unlikely that perceptual representations would activate each other, although it is not inconceivable that semantic associations might lead to orthographic associations.

We assume that the activation of cues in STM decays in the interval between trials (Altmann, 2002; Altmann \& Gray, 2002). When a cue is presented on trial $n$, there will be residual activation for cues from trial $n-1$. In the race between the comparisons to short- and long-term memory described earlier, residual activation from trial $n-1$ for the cue presented on trial $n$ will facilitate the comparison to STM. The perceptual representation of the presented cue will match a decayed trace in STM, priming that comparison process (i.e., increasing the speed of that runner in the race). Stronger residual activation leads to more priming, resulting in faster cue encoding.

Differences among transitions arise from differences in residual activation that prime cue encoding. For example, assume $O D D$ was the cue on trial $n-1$. When a cue is presented on trial $n$, there will be strong residual activation for $O D D$, intermediate residual activation for $E V E N$, and little or no residual activation for $H I G H$

\footnotetext{
${ }^{1}$ The proportion of subjects who produce a target word in response to a cue word can be interpreted as a measure of the associative strength between the two words. The free association norms compiled by Nelson et al. (1999) list the following production proportions (as cue-target pairs) for the words used in the present study: $O D D-E V E N=.558, E V E N-O D D=$ $.621, H I G H-L O W=.655$, and $L O W-H I G H=.777$. Each target was the most frequent free associate to the cue, suggesting strong associations. The remaining pairs (e.g., $O D D-H I G H$ ) never occurred together by free association and can be considered to have much weaker associations.
} 
and $L O W$. These different amounts of residual activation will affect the rate of comparison to STM in the race to encode the cue on trial $n$. If the cue on trial $n$ is $O D D$ (i.e., a cue repetition), cue encoding will benefit from strong residual activation because the rate of comparison to STM will be increased. Cue encoding will be fast due to repetition priming. If the cue on trial $n$ is $E V E N$ (i.e., a task repetition), cue encoding will benefit from intermediate residual activation because the rate of comparison to STM will be increased, but to a lesser extent than for cue repetitions because of less residual activation. Cue encoding will be fast due to associative priming. If the cue on trial $n$ is $H I G H$ or $L O W$ (i.e., a task alternation), cue encoding will not benefit much from little or no residual activation because the rate of comparison to STM will be essentially unchanged. Cue encoding will be slow due to a lack of priming. Cue encoding time is a substantial component of RT except at long SOAs, so different rates of cue encoding can produce differences in RT. It follows that differences among transitions emerge from differential priming of cue encoding. Cue repetitions are faster than task repetitions (i.e., a repeated cue encoding benefit) because repetition priming is greater than associative priming of cue encoding. Task repetitions are faster than task alternations (i.e., a "switch cost") because associative priming is greater than no priming of cue encoding.

Compound cue retrieval. Once a cue and a target have been encoded, a response has to be selected. We propose that the cue and the target combine as a compound cue for retrieving response categories from long-term memory (Dosher \& Rosedale, 1989; Logan \& Bundesen, 2003, 2004; Ratcliff \& McKoon, 1988). The cue and the target will retrieve response categories from long-term memory with different strengths based on preexisting associations between response categories (as described above) and between targets and response categories. We assume that subjects already know whether a target is odd or even and high or low with respect to 5 ; none of the subjects in our experiments lacked such knowledge.

Response selection is based on the multiplicative combination of the strengths for each response category (cf. Gillund \& Shiffrin, 1984; Hintzman, 1986; Raaijmakers \& Shiffrin, 1981). When the cue and the target are both strongly associated with the same response category (e.g., $O D D$ and 3 with $o d d$ ), the product of their strengths will be large, resulting in a high probability of retrieving that response category. When the cue and the target are weakly associated or unassociated with the same response category (e.g., $O D D$ and 3 with high), the product of their strengths will be small, resulting in a low probability of retrieving that response category. Intermediate products can occur when either the cue or the target is strongly or partially associated with a response category (e.g., $O D D$ and 3 with even).

Differences in cue-target congruency are predicted to arise from the differences among products. For congruent trials (e.g., $O D D$ $3)$, the largest product exists for the correct response category $(o d d)$, resulting in a high probability of retrieving that response category. For incongruent trials (e.g., $E V E N-3$ ), the largest product also exists for the correct response category $(o d d)$ because the cue is partially associated with that response category and the target is strongly associated with that response category. The probability of retrieving the correct response category will not be as high as for congruent trials because of the difference in associative strength with the cue, but it will be higher relative to the probabilities of retrieving incorrect response categories. A high probability for retrieving the correct response category leads to a fast RT and high accuracy. The differences in retrieval probabilities between congruent and incongruent trials predict a cue-target congruency effect: Congruent trials will be faster and more accurate than incongruent trials. Furthermore, our priming account implies a close relationship between cue-target congruency and priming of cue encoding because both effects rely on the same strengths of association between response categories. This relationship allows us to model these effects using the same parameters, as described below.

Summary. Our priming account explains differences among transitions with differential priming of cue encoding and differences in cue-target congruency with different probabilities of retrieving the correct response category by compound cue retrieval. No executive control process such as task-set reconfiguration has to be invoked to switch tasks. Moreover, there is no need to invoke different task sets or to switch task sets for task alternations. There is only a single task set that permits flexible performance of either task because the underlying mechanisms of encoding and retrieval are identical for both tasks. This task set involves encoding the cue, encoding the target, and responding with what is retrieved from memory via compound cue retrieval.

\section{The Priming Model}

A criticism of task-set reconfiguration is that it tends to be underspecified (Logan \& Gordon, 2001). How does task-set reconfiguration occur? To avoid a similar criticism of our priming account, we formalized our mechanisms of cue encoding and compound cue retrieval, first as a computer simulation and then as a mathematical model. To avoid redundancy, we describe only the mathematical model and its fits to experimental data.

Cue encoding. Following Logan and Bundesen (2003, 2004; also see Arrington \& Logan, 2004), we assume that RT on each trial is the sum of cue encoding and residual processing times. Response selection time is separate from residual processing time in the priming model, but to simplify our exposition at this point, we allow response selection to be subsumed in residual processing. The contribution of cue encoding time to RT is assumed to vary as a function of SOA, yielding a time-course function. At SOA $=0$, when the cue has not been encoded prior to target onset,

$$
R T=R T_{\mathrm{Base}}+\mu,
$$

where $R T_{\text {Base }}$ is residual processing time (which includes target encoding and response execution) and $\mu$ is mean cue encoding time. At a long SOA, when the cue has been encoded prior to target onset,

$$
R T=R T_{\text {Base }}
$$

Cue encoding time is assumed to be exponentially distributed. An advantage of exponential distributions is that they involve a single parameter representing the rate of processing, making them mathematically tractable for modeling purposes. For this reason, exponential distributions are found in many formal models of cognitive processing (Townsend \& Ashby, 1983). We assume that the probability that a cue has been encoded during a particular SOA is determined by the cumulative distribution of cue encoding 
times. RT is determined by Equation 1 with a probability of $\exp [-S O A / \mu]$ and by Equation 2 with a probability of 1 $\exp [-S O A / \mu]$. When these probabilities are combined (see Logan \& Bundesen, 2003),

$$
R T=R T_{\text {Base }}+\mu \cdot \exp [-S O A / \mu] .
$$

Equation 3 allows the priming model to capture the time-course function observed in explicitly cued performance. As SOA increases, the contribution of cue encoding time to RT decreases, resulting in a faster RT. We assume that residual processing time is constant across conditions.

Following Logan and Bundesen (2003, p. 578), mean cue encoding time $\mu$ for each transition is based on a race between two comparison processes: $v_{\mathrm{STM}}$, the rate of comparison of the presented cue to its transient representation in STM, and $v_{\mathrm{LTM}}$, the rate of comparison of the presented cue to its permanent representation in long-term memory. We assume that the times required for the comparisons to short- and long-term memory are both exponentially distributed. Consequently, the distribution of finishing times for the race between the comparison processes will also be exponentially distributed with a rate parameter that is equal to the sum of the rate parameters for the runners in the race (i.e., $v_{\mathrm{STM}}+$ $\left.v_{\text {LTM }}\right)$. Thus, mean cue encoding time is as follows:

$$
\mu=\frac{1}{\nu_{\mathrm{STM}}+\nu_{\mathrm{LTM}}} .
$$

The presented cue has been encoded when it matches either a short- or long-term memory representation, with cue encoding time determined by the comparison process that finishes first. As the strength of the representation of the presented cue in STM increases, $v_{\mathrm{STM}}$ increases and $\mu$ decreases. There are separate values of $\mu$ for cue repetitions, task repetitions, and task alternations due to differences in $v_{\mathrm{STM}}$ that are described below.

The critical factor influencing $v_{\mathrm{STM}}$ is residual activation from the preceding trial for the presented cue. For presented cue $x, v_{\mathrm{STM}}$ is calculated as the strength of evidence $\eta$ for response category $i$ associated with cue $x$ from the preceding trial multiplied by an STM decay factor $d$ :

$$
\nu_{\mathrm{STM}}=\eta(i \mid x) \cdot d .
$$

Strong residual activation for the presented cue (i.e., a large $\eta$ value that has decayed) results in a faster rate of comparison to STM (Equation 5), leading to faster cue encoding (Equation 4). The present formulation of the priming model only involves residual activation from the immediately preceding trial. It could be extended to deal with residual activation from multiple preceding trials by including power-function decay of cues across trials; this extension is considered in the context of long-term priming in the General Discussion section. STM decay is a constant in Equation 5 because the time between the response on the preceding trial and the onset of the cue on the present trial is a constant in our experiments. We assume that decay occurs during the interval when no cue is available for processing, not during the SOA when the presented cue is being encoded.

Differences in residual activation arise from differences in the activation of cues from the preceding trial. Recall that if the presented cue is $O D D$, odd and even will be retrieved, and we assume that EVEN becomes partially activated in STM. For sim- plicity, we assume that the activation of cues in STM corresponds to $\eta$ values reflecting the strengths of associations between cues and response categories in long-term memory: $\eta_{\mathrm{P}}$ is the evidence for the response category strongly associated with the presented cue, $\eta_{\mathrm{A}}$ is the evidence for the response category partially associated with the presented cue based on associations between response categories in long-term memory, and $\eta_{U}$ is the evidence for the response category unassociated with the presented cue. Stronger associations imply stronger evidence for a response category (or activation of a cue); it follows that $\eta_{\mathrm{P}}>\eta_{\mathrm{A}}>\eta_{\mathrm{U}}$.

Residual activation for the presented cue depends on the $\eta$ value from the preceding trial for the presented cue (Equation 5). Differences in priming of cue encoding across transitions occur because of different $\eta$ values: $\eta_{\mathrm{P}}$ when the cue repeats, $\eta_{\mathrm{A}}$ when the cue changes and is associated with the preceding cue, or $\eta_{\mathrm{U}}$ when the cue changes and is unassociated with the preceding cue. It follows that $v_{\mathrm{STM}}$ will be high for cue repetitions, intermediate for task repetitions, and low for task alternations, resulting in mean cue encoding times (Equation 4) that are fast, intermediate, and slow, respectively. Differences among transitions (repeated cue encoding benefits and "switch costs") arise from differential priming of cue encoding in the priming model.

Differences among transitions decrease with SOA because priming affects cue encoding and the contribution of cue encoding to RT decreases with SOA, leading to smaller differences among transitions at long SOAs. In the priming model, the reduction in "switch cost" with preparation time is due to a reduction in the contribution of cue encoding to RT, not an executive control process such as task-set reconfiguration. At an infinite SOA, the priming model predicts no differences among transitions (i.e., no residual switch costs) because cue encoding will no longer contribute to RT; we discuss this aspect of the model in more detail in the General Discussion section.

Compound cue retrieval. Once a cue and a target have been encoded, they have to be categorized and a response has to be selected. We assume that the same $\eta$ values used in cue encoding are used in compound cue retrieval. These $\eta$ values determine the rates of retrieving different categorizations of cues and targets from long-term memory; larger $\eta$ values lead to faster retrieval rates. The probability $P$ of retrieving response category $i$, given cue $x$ and target $y$, is the ratio of the retrieval rate $v$ for categorizations of $i$ to the sum of the retrieval rates for categorizations of $j$, where $j$ is any of the response categories in the response set $R$ :

$$
P(i \mid x, y)=\frac{\nu(i \mid x, y)}{\sum_{j \in R} \nu(j \mid x, y)} .
$$

Equation 6 is the Shepard-Luce choice rule (Luce, 1959, 1963; Shepard, 1957), which is a core component in many models of cognition (see Logan, 2004). The rate parameter $v$ is derived from the product of the evidence $\eta$ that cue $x$ and target $y$ are associated with response category $i$ and the bias $\beta$ toward selecting response category $i$ :

$$
\nu(i \mid x, y)=\eta(i \mid x, y) \beta_{i} .
$$

The bias $\beta$ in Equation 7 is a top-down parameter determined by the subject (Logan \& Gordon, 2001). We assume an equal bias for all response categories and set $\beta$ to 1.0. Additional top-down 
parameters related to the categorization of stimuli from multiobject displays (e.g., attention weights and priority parameters, as in Bundesen, 1990; Logan \& Gordon, 2001) have been omitted from Equation 7 because they are also assumed to be 1.0 ; there is only one cue and one target presented on each trial. In this regard, the priming model differs from other models of task switching in that there is no differential top-down input on task repetition and task alternation trials (Gilbert \& Shallice, 2002; Yeung \& Monsell, 2003). Consequently, $\eta$ can be substituted directly for $v$ in Equation 6 , such that the probability of retrieving a response category is based solely on bottom-up input:

$$
P(i \mid x, y)=\frac{\eta(i \mid x, y)}{\sum_{j \in R} \eta(j \mid x, y)} .
$$

The $\eta$ value for each response category in Equation 8 is determined jointly by the cue and the target. This joint evidence is the product of the evidence $\eta$ for response category $i$ given cue $x$ and the sum of the evidence $\eta$ for response category $i$ given target $y$, where $y$ belongs to the target stimulus set $S$ associated with response category $i$ :

$$
\eta(i \mid x, y)=\eta(i \mid x) \cdot \sum_{y \in S_{i}} \eta(i \mid y) .
$$

Equation 9 represents compound cue retrieval: Evidence from the cue and the target combine multiplicatively to retrieve response categories from long-term memory (Ratcliff \& McKoon, 1988). Multiplicative combination of evidence from multiple sources is a central component of many models of memory retrieval (e.g., Gillund \& Shiffrin, 1984; Hintzman, 1986; Raaijmakers \& Shiffrin, 1981), but we note that the multiplicative combination in the priming model does not reflect a gestalt or configural representation of the cue and the target. Instead, the cue and the target are considered to be separate inputs that converge on a common response category; evidence for that response category is only available if both inputs are present simultaneously. In the context of Equation 9, if the target has yet to be encoded, the evidence from that input is zero, which makes the combined evidence for the response category equal to zero. Summation of evidence for all targets associated with a response category is not essential for our present model fits, but it provides a framework for the priming model to interface with models of categorization that deal with similarity among targets (e.g., Nosofsky, 1986; Nosofsky \& Palmeri, 1997). For example, if the target set consisted of items that differed in similarity, then a target could provide some evidence for multiple, similar targets. Summation of evidence for all targets would allow the similarity within the target set to influence compound cue retrieval.

For the experiments reported below, two response categories were mapped onto each response key. The probability of selecting response key $A_{n}$ (where $n \in\{1,2\}$ for the two response keys) is the sum of the retrieval probabilities (from Equation 8) of the response categories $i$ that are assigned to that response key:

$$
P\left(A_{n} \mid x, y\right)=\sum_{i \in A_{n}} P(i \mid x, y) .
$$

Note that Equation 10 involves experimentally defined associations between unassociated response categories at a conceptual level. We assume that subjects have to maintain spatial mappings between selected responses and physical response keys to execute their responses.

The preceding equations describe how response categories are retrieved but not how responses are selected. We assume that evidence (Equation 9) and probabilities (Equations 8 and 10) associated with response categories are used to select responses based on Nosofsky and Palmeri's (1997) exemplar-based random walk model. Other authors have also suggested or used random walks for compound cue retrieval (Dosher \& Rosedale, 1989; Ratcliff \& McKoon, 1988).

In random walk models, response categories are retrieved from long-term memory and accumulate over time in separate response counters until a criterion is reached (for an overview, see Ratcliff, 2001). Response counters are incremented at discrete time intervals or steps of the random walk (cf. continuous accumulation as in diffusion models; Ratcliff, 1978) until the difference between the counters meets a response criterion $K$, at which point the response associated with the higher counter is selected. The value of $K$ is determined by the subject, not by the stimuli (Logan \& Gordon, 2001). We assume a fixed value of $K$, making it a constant in all equations.

The drift rate of the random walk-the rate of evidence accumulation for a specific response category-is the ratio of the amount of evidence for that response category to the summed evidence for all response categories (Equation 8, although our implementation of a two-choice random walk involves Equation 10). A high probability of retrieving a response category produces a fast drift rate, whereas a low probability produces a slow drift rate.

Different response categorizations race against one another and each step of the random walk is determined by whichever categorization has the fastest finishing time. This process continues until the random walk meets the response criterion. We assume each response categorization has an exponentially distributed finishing time and that the finishing times for the race are exponentially distributed with a rate equal to the sum of the retrieval rates for each response categorization. Consequently, the time per step of the random walk, $R T_{\text {Step }}$, is the reciprocal of the sum of the retrieval rates for all response categories $j$, given cue $x$ and target $y$ (Nosofsky \& Palmeri, 1997; also see Logan, 2002; Logan \& Gordon, 2001). This value is equivalent to the denominator of Equation 8:

$$
R T_{\text {Step }}(x, y)=\frac{1}{\sum_{j \in R} \eta(j \mid x, y)} .
$$

The number of steps for the random walk to terminate, $N_{\text {Step }}$, given cue $x$ and target $y$, is calculated from the probabilities from Equation 10 (which correspond to drift rates) and the response criterion $K$, where we assume that $K$ does not differ among responses. Consequently, the number of steps is determined by the following set of equations derived by Busemeyer (1982) and adapted from Nosofsky and Palmeri (1997):

$$
\begin{array}{r}
N_{\text {Step }}(x, y)=\frac{1}{P\left(A_{1} \mid x, y\right)-P\left(A_{2} \mid x, y\right)}\left[\theta_{1}(2 K)-\theta_{2}(K)\right], \\
\text { if } P\left(A_{1} \mid x, y\right) \neq P\left(A_{2} \mid x, y\right),
\end{array}
$$


where

$$
\begin{aligned}
\theta_{1} & =\frac{\left[P\left(A_{1} \mid x, y\right) / P\left(A_{2} \mid x, y\right)\right]^{2 K}+1}{\left[P\left(A_{1} \mid x, y\right) / P\left(A_{2} \mid x, y\right)\right]^{2 K}-1} \text { and } \\
\theta_{2} & =\frac{\left[P\left(A_{1} \mid x, y\right) / P\left(A_{2} \mid x, y\right)\right]^{K}+1}{\left[P\left(A_{1} \mid x, y\right) / P\left(A_{2} \mid x, y\right)\right]^{K}-1} .
\end{aligned}
$$

Mean compound cue retrieval time (i.e., response selection time), $R T_{\mathrm{CCR}}$, is equal to the mean time for the random walk to finish with cue $x$ and target $y$, which is the product of the time per step (Equation 11) and the number of steps (Equation 12):

$$
R T_{\mathrm{CCR}}(x, y)=R T_{\mathrm{Step}}(x, y) \cdot N_{\text {Step }}(x, y) .
$$

The probability that the random walk selected the correct response key $A_{c}$ (if response key $A_{1}$ is associated with the correct response category) is given by the equation (Nosofsky \& Palmeri, 1997)

$$
\begin{aligned}
P\left(A_{c} \mid x, y\right)=\frac{1-\left[P\left(A_{2} \mid x, y\right) / P\left(A_{1} \mid x, y\right)\right]^{K}}{1-\left[P\left(A_{2} \mid x, y\right) / P\left(A_{1} \mid x, y\right)\right]^{2 \mathrm{~K}}}, & \\
& \text { if } P\left(A_{1} \mid x, y\right) \neq P\left(A_{2} \mid x, y\right) .
\end{aligned}
$$

Compound cue retrieval time is separate from residual processing time, so Equation 3 can be amended to calculate RT:

$$
R T=R T_{\mathrm{Base}}+R T_{\mathrm{CCR}}+\mu \cdot \exp [-S O A / \mu] .
$$

Differences in cue-target congruency occur because of different $\eta$ values across response categories in compound cue retrieval. On congruent trials (e.g., $O D D-3$, where the correct response is $o d d$ ), $\eta($ odd $\mid O D D, 3)$ will be high because both $\eta($ odd $\mid O D D)$ and $\eta(o d d \mid 3)$ will be equal to $\eta_{\mathrm{P}}$ (see Equation 9). The probability of correctly selecting odd as a response will be high, resulting in high accuracy, and the drift rate of the random walk will be fast, resulting in a fast compound cue retrieval time. On incongruent trials (e.g., EVEN-3, where the correct response is odd), $\eta$ (odd $E V E N, 3$ ) will be relatively high (but not as high as on congruent trials) because $\eta($ odd $\mid E V E N)$ will be equal to $\eta_{\mathrm{A}}$ and $\eta($ odd $\mid 3)$ will be equal to $\eta_{\mathrm{P}}$. Evidence for the corresponding response category, $\eta($ even $\mid E V E N, 3)$, will be lower, however, because $\eta($ even $\mid E V E N)$ will be equal to $\eta_{\mathrm{P}}$ but $\eta($ even $\mid 3)$ will be equal to $\eta_{\mathrm{U}}$. When evidence from the cue and the target is combined multiplicatively in Equation 9, there will be greater evidence for odd than even, but compared with congruent trials, the probability of correctly selecting odd as a response will be slightly lower, resulting in lower accuracy, and the drift rate of the random walk will be slower, resulting in a slower compound cue retrieval time. The differences in RT and accuracy between incongruent and congruent trials represent the cue-target congruency effect. Sample calculations for producing the cue-target congruency effect are available as an online supplement (http://dx.doi.org/10.1037/ 0096-3445.134.3.343.supp).

Summary. The priming model instantiates the assumptions and mechanisms in our priming account to explain differences among transitions and cue-target congruency. As noted earlier, the same evidence parameters ( $\eta$ values) are used to capture differences in priming of cue encoding and select responses via compound cue retrieval. The ability of the priming model to fit transition data is constrained by its ability to fit cue-target congruency data, and vice versa. Evidence parameters cannot be changed arbitrarily to fit differences among transitions without affecting the fit to differences in cue-target congruency. This constraint is important because it links priming of cue encoding and compound cue retrieval to the same representations of bottom-up input within a common framework.

The equations and assumptions in the preceding sections form the basis of the priming model. One modification is made below to account for an interaction between transition and cue-target congruency observed in our experiments. None of the equations, assumptions, subsequent modifications, or proposed extensions to the priming model include executive control processes such as task-set reconfiguration. If the priming model can account for our experimental data, then the idea that task sets have to be reconfigured when task switching occurs is questionable.

\section{General Method}

All experiments were based on the method described below. Modifications of the method for Experiments 2 and 3 are noted in subsequent sections.

\section{Subjects}

A total of 84 undergraduate psychology students from Vanderbilt University participated in exchange for partial course credit. Each experiment involved a different group of 28 subjects.

\section{Apparatus and Stimuli}

The experiments were conducted using E-Prime software (Version 1.1; Psychology Software Tools, 2002) operating on Dell Dimension computers. Stimuli were displayed on Sony Trinitron monitors, and responses were recorded from standard QWERTY keyboards. All experiments included the cue words $O D D, E V E N, H I G H$, and LOW. Experiments 2 and 3 also included dual cues, which were unions of the single-word taskspecific cues (e.g., ODD-EVEN and HIGH-LOW). The targets were the digits $1,2,3,4,6,7,8$, and 9 . Cues and targets were presented in a white, 18-point Verdana font on a black background and were 1-cm tall. Stimulus widths were $3.3 \mathrm{~cm}$ (ODD and $L O W$ cues), $3.8 \mathrm{~cm}$ (EVEN and $H I G H$ cues), and $0.7 \mathrm{~cm}$ (targets). Viewing distance was approximately $60 \mathrm{~cm}$, a distance at which $1 \mathrm{~cm}$ of the display subtended about $1^{\circ}$ of visual angle. The same set of SOAs $(0,100,200,400$, and $800 \mathrm{~ms})$ was used in each experiment.

\section{Procedure}

Subjects were seated individually in private testing rooms after providing informed consent. Instructions were presented on screen and read by subjects at their own pace. The instructions described the format of each trial, the nature of the tasks, and the response-key mappings. Subjects were instructed to make their responses as quickly and accurately as possible, basing their responses on the target in the context of the cued task. Subjects were not instructed to treat the cue and the target as a compound cue. To clarify the relationship between the cues and the targets, the instructions included the following sentence: "Please note that the cue word only indicates the task to be performed and will not necessarily match the correct response." This sentence was included to dissuade subjects from responding solely to the cue and ignoring the target, particularly on incongruent trials. To emphasize this point, the experimenter gave instructions for how to respond to the incongruent cue-target combinations $O D D-4$ and $H I G H-2$. Following the instructions, subjects had the opportunity to ask the experimenter any questions about the procedure. 
Each experiment was divided into blocks of 160 trials (Experiment 1) or 120 trials (Experiments 2 and 3). There were 960 trials in total in each experiment. A full replication of all combinations of cues, targets, and SOAs occurred every block, two blocks, or four blocks for Experiments 1, 2, and 3, respectively. Blocks were separated by self-paced rest periods. Cues, targets, and SOAs appeared in a random order within each block for each individual subject.

Each trial began with a fixation display consisting of a plus sign in the center of the screen for 1,000 ms. The screen was then cleared, and a cue was displayed, centered one line above the position previously occupied by the plus sign. After a variable SOA, a target was displayed, centered two lines below the cue. The cue and the target remained on screen until the subject made a response, then the next trial commenced immediately. Responses were assigned to the $C$ and $M$ keys for each task, and all possible response-key mappings were counterbalanced across subjects within each experiment.

\section{Data Trimming and Classification}

A few subjects in each experiment with mean accuracy below $90 \%$ or mean RT exceeding 2.5 standard deviations of the group mean were replaced. An accuracy criterion was implemented to ensure a high level of performance and that a sufficient number of trials would contribute to each subject's mean RTs, especially in Experiment 3.

The first trial of each block and trials with RT exceeding 3,000 ms ( $M=$ $4.3 \%$ of all trials across experiments) were excluded from the analyses. Trials with incorrect responses were excluded from all RT analyses. The transition for each trial was classified post hoc based on its relationship to the preceding trial as a cue repetition, task repetition, or task alternation. Cue-target congruency was classified post hoc based on whether the cue and target for each trial were directly associated with the same response category (congruent) or different response categories (incongruent).

\section{Data Analysis}

The RT and accuracy data for each experiment were submitted to separate repeated-measures analyses of variance (ANOVAs), with the results summarized in tables. Planned comparisons were conducted using relevant error terms from the omnibus ANOVAs and are reported in the text. For all statistical analyses, the reported $\eta^{2}$ represents the partial $\eta^{2}$ effect size statistic and is unrelated to $\eta$ in the priming model.

\section{Experiment 1}

The goal of the first experiment was to evaluate the sufficiency of our priming account. We expected a difference between cue repetitions and task repetitions, reflecting faster cue encoding for cue repetitions (i.e., greater repetition priming than associative priming). We expected a difference between task repetitions and task alternations, reflecting faster cue encoding for task repetitions (i.e., greater associative priming than no priming). The contribution of these differences in cue encoding to RT were predicted to decrease with SOA, leading to a reduction in the differences among transitions with increased preparation time (characterized by a Transition $\times$ SOA interaction). We also expected a cue-target congruency effect, whereby incongruent trials would be slower and less accurate than congruent trials.

\section{Method}

Experiment 1 was conducted according to the description given in the General Method section, with the words $O D D$ and EVEN cuing odd-even judgments and the words $H I G H$ and $L O W$ cuing high-low judgments of single-digit targets, with five SOAs.

\section{Results and Discussion}

Mean RT and accuracy (as percentage of correct responses) were calculated for each subject for each combination of transition, cue-target congruency, and SOA; these data are presented in Appendix A. Accuracy was high and within-subject correlations between RT and accuracy did not indicate any speed-accuracy trade-offs; therefore, the analyses focused on RT.

RT analysis. Mean RTs across subjects for each transition as a function of SOA are presented in the top panel of Figure 1. Mean RT for cue repetitions (914 ms) was faster than task repetitions $(1,036 \mathrm{~ms})$, which in turn was faster than task alternations $(1,104$ $\mathrm{ms}$ ). The large repeated cue encoding benefit replicates previous research using multiple cues per task (Arrington \& Logan, 2004; Brass \& von Cramon, 2004; Logan \& Bundesen, 2003, 2004; Mayr \& Kliegl, 2003). The sizable switch cost is consistent with some results (e.g., Mayr \& Kliegl, 2003) but not others (e.g., Logan \& Bundesen, 2003), although we demonstrate in a later section that the magnitude of the "switch cost" depends on the amount of priming of cue encoding. Mean RT and the differences among transitions decreased as SOA increased (see Figure 1), replicating the reduction in switch cost with preparation time (e.g., Meiran, 1996).

Mean RTs partitioned by transition and cue-target congruency are presented in the top row of Figure 2. There was the predicted cue-target congruency effect-mean RT for incongruent trials (1,074 ms) was slower than for congruent trials (962 ms) - but cue-target congruency interacted with transition (see Figure 2): The cue-target congruency effect was smaller for cue repetitions (53 ms) than task repetitions (147 ms) and task alternations (136 ms), which did not differ.

These observations were supported by a 3 (transition: cue repetition, task repetition, or task alternation) $\times 2$ (cue-target congruency: congruent or incongruent) $\times 5$ (SOA: 0, 100, 200, 400, or $800 \mathrm{~ms}$ ) repeated-measures ANOVA, with the results summarized in Table 1. Nonorthogonal comparisons indicated that mean RT for cue repetitions was faster than for task repetitions, $F(1$, $54)=77.59, p<.01$, which in turn was faster than for task alternations, $F(1,54)=23.42, p<.01$. Orthogonal comparisons revealed that the cue-target congruency effect was smaller for cue repetitions than for the mean of task repetitions and task alternations, $F(1,54)=38.98, p<.01$, which did not differ, $F(1,54)<1$.

Further analysis of the RT data suggested that the interaction between transition and cue-target congruency was related to the cue-target congruency from the preceding trial. Mean RTs were calculated for each subject based on the transition and cue-target congruency on trial $n$ with respect to the cue-target congruency on trial $n-1$; these data are depicted as cue-target congruency effects for trial $n$ in the top panel of Figure 3. The critical effect in these data was a three-way interaction: The cue-target congruency effect on trial $n$ differed for cue repetitions (compared with task repetitions and task alternations) based on the cue-target congruency of trial $n-1$. As is evident in Figure 3, the cue-target congruency effect was essentially eliminated when the preceding trial was incongruent for cue repetitions.

These observations were supported by a 3 (transition: cue repetition, task repetition, or task alternation) $\times 2$ (cue-target con- 

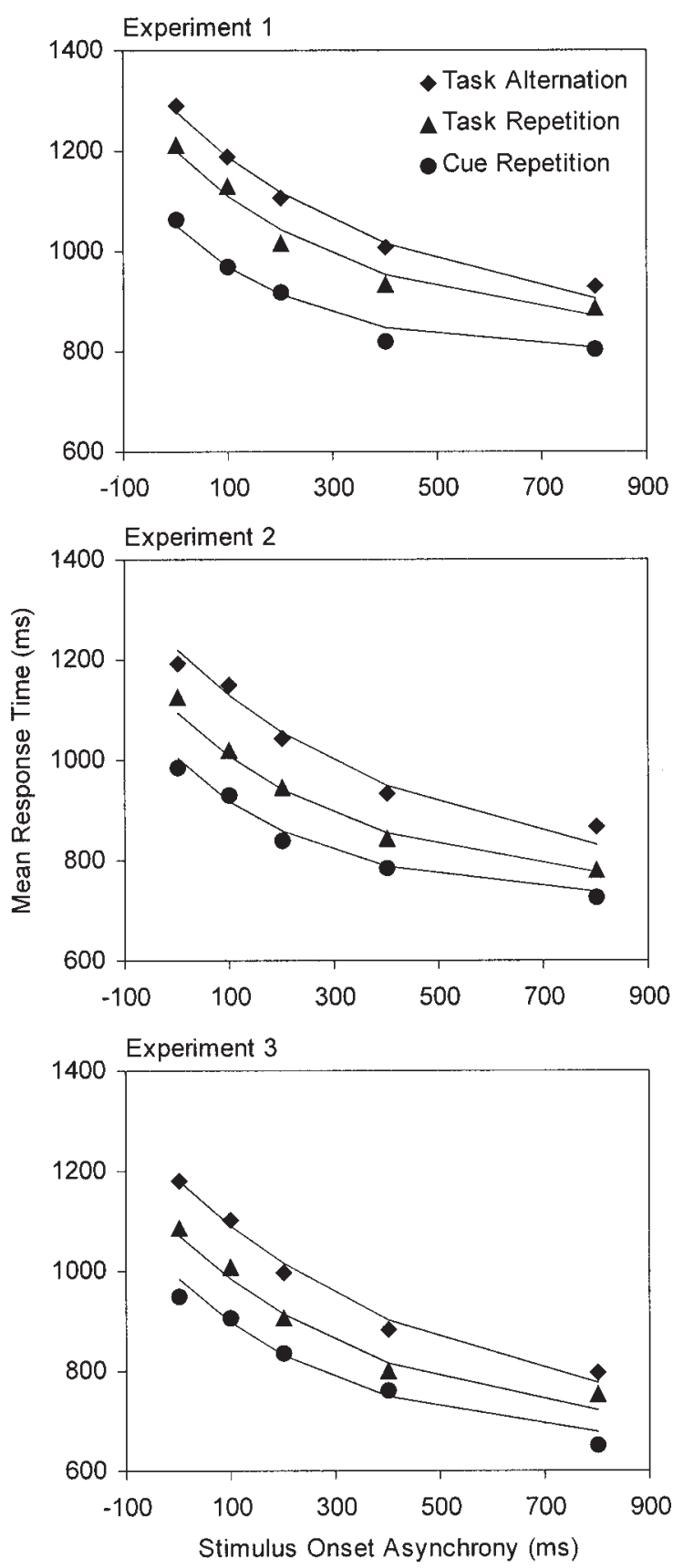

Figure 1. Mean response times for task alternations, task repetitions, and cue repetitions as a function of stimulus onset asynchrony for Experiments 1,2 , and 3. Points represent observed data, and lines represent model predictions.

gruency on trial $n$ : incongruent or congruent) $\times 2$ (cue-target congruency on trial $n-1$ : incongruent or congruent) repeatedmeasures ANOVA, collapsing across SOA and excluding the first two trials of each block. To avoid redundancy, we report only statistics associated with cue-target congruency on trial $n-1$. There was a main effect of cue-target congruency on trial $n-1$, $F(1,27)=18.59, M S E=3,714.03, p<.01, \eta^{2}=.41$, and it interacted with transition, $F(2,54)=12.74, M S E=4,273.13, p<$ $.01, \eta^{2}=.32$, and cue-target congruency on trial $n, F(1,27)=$ 5.42, $M S E=6,028.01, p<.05, \eta^{2}=.17$. The three-way interaction was also significant, $F(2,54)=7.83, M S E=3,689.82, p<$ $.01, \eta^{2}=.23$. A three-way interaction contrast comparing the differences in cue-target congruency on trial $n$ by the cue-target congruency on trial $n-1$ for cue repetitions to the mean of those differences for task repetitions and task alternations was significant, $F(1,54)=13.15, p<.01$.

To understand the interaction between transition and cue-target congruency, we considered how cues might retrieve response categories not only from long-term memory but also from STM. After response selection, a temporary association between the cue and the selected response category might be stored in STM. These cue-response associations could be viewed as instances (Logan, 1988) that are available for only a limited time in STM because of the dynamic relationship between cues and responses based on cue-target congruency. The transient nature of these cue-response associations differentiates them from the long-term stimulusresponse associations in task-set priming accounts, although we believe both types of associations could coexist as discussed later. For simplicity, we assume these cue-response associations hold only from one trial to the next before they rapidly decay, unless the cue involved in the association reappears to maintain the temporary association. If the association is maintained, then there would be strong residual evidence for that response category and response selection would be affected. The situation in which these temporary associations could affect performance is on cue repetition trials, where the cue can retrieve the response category it helped select on the preceding trial.

The interaction between transition and cue-target congruency would occur as follows: If trial $n$ is a cue repetition, strong evidence for the response category selected on trial $n-1$ would be used to select a response based on the temporary cue-response association. If trials $n-1$ and $n$ were both congruent (e.g., $O D D-3$ and $O D D-7)$, there would be strong evidence for only the correct response category $(o d d)$. If trial $n-1$ was congruent $(O D D-3)$ and trial $n$ was incongruent $(O D D-4)$, there would be strong evidence from the cue for only the incorrect response category (odd, because trial $n$ requires an even response). Consequently, if trial $n-1$ was congruent, a cue-target congruency effect would be expected on trial $n$ because of the discrepancy in evidence for the correct response category (i.e., strong evidence for the correct response category when trials $n-1$ and $n$ are both congruent; strong evidence for the incorrect response category when trial $n-1$ is congruent and trial $n$ is incongruent). If trials $n-1$ and $n$ were both incongruent ( $O D D-4$ and $O D D-6)$, there would be strong evidence for the correct and incorrect response categories; the correct response category (even) would have strong evidence from the temporary cue-response association, whereas the incorrect response category (odd) would have strong evidence from the presented cue accessing long-term memory. If trial $n-1$ was incongruent $(O D D-4)$ and trial $n$ was congruent $(O D D-3)$, there would also be strong evidence for the correct and incorrect response categories; the incorrect response category (even) would have strong evidence from the temporary cue-response association, whereas the correct response category (odd) would have strong evidence from the presented cue. Consequently, if trial $n-1$ was incongruent, a cue-target congruency effect would not be expected 


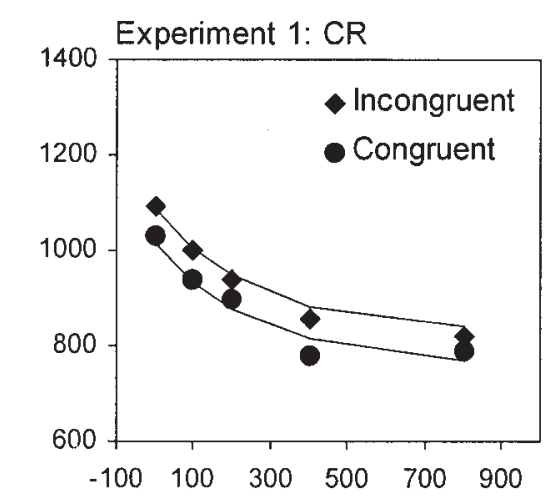

Experiment 1: TR
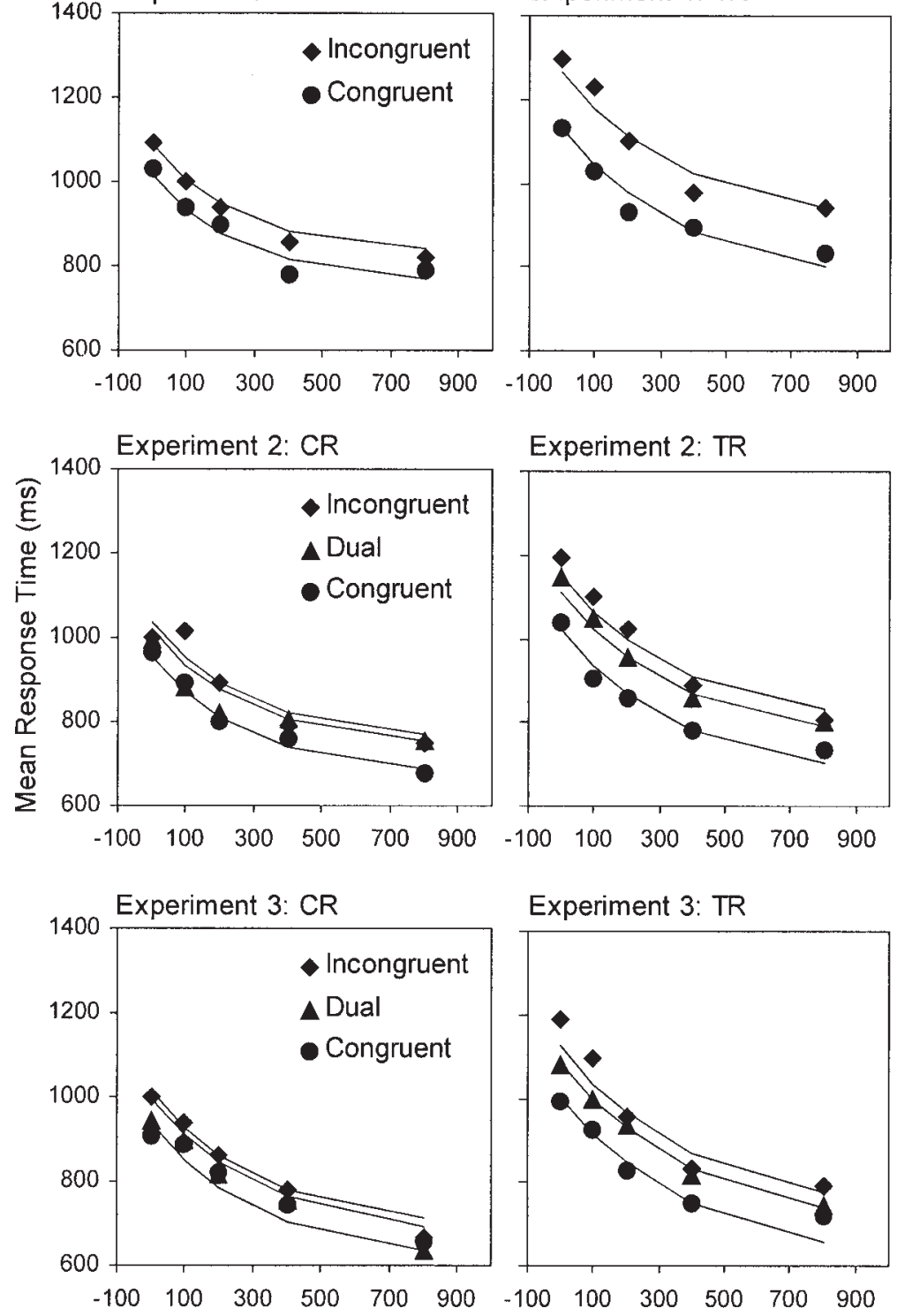

Experiment 2: TR



Experiment 2: TA

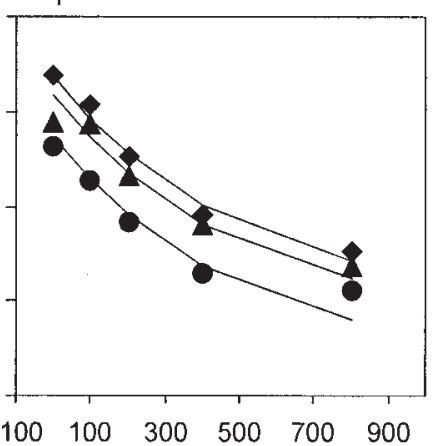

Experiment 3: TR

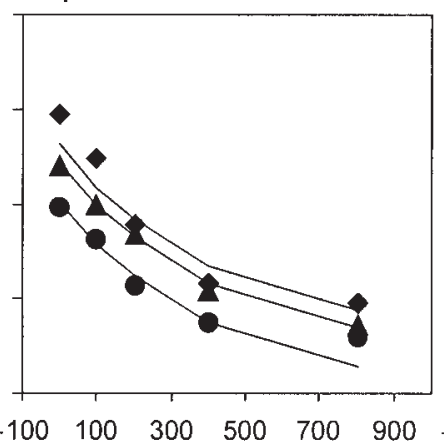

Experiment 3: TA

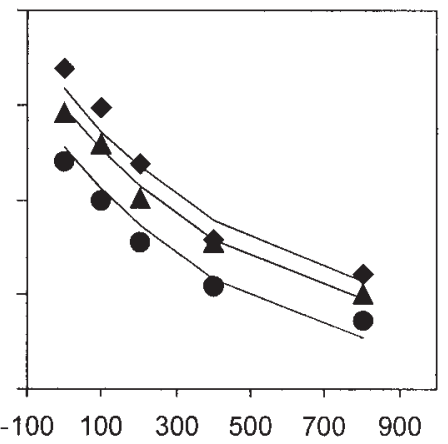

Stimulus Onset Asynchrony (ms)

Figure 2. Mean response times for incongruent, dual, and congruent trials for cue repetitions (CR), task repetitions (TR), and task alternations (TA) as a function of stimulus onset asynchrony for Experiments 1, 2, and 3. Points represent observed data, and lines represent model predictions. The ordinal pattern of model predictions corresponds to the observed data for all conditions, except for a reversal of incongruent and dual trials for CRs in Experiments 2 and 3.

because there is equal evidence for the correct and incorrect response categories, regardless of the cue-target congruency of trial $n$. This hypothesis about the interaction is simple in principle and accounts qualitatively for the interaction in Figure 3.

Accuracy analysis. Overall accuracy across subjects was high ( $M=96.4 \%$; see Appendix A). Mean accuracy decreased from cue repetitions $(97.3 \%)$ to task repetitions $(96.7 \%)$ to task alternations $(95.9 \%)$. Mean accuracy was lower for incongruent trials $(95.5 \%)$ than congruent trials $(97.8 \%)$ as predicted by our priming account. Cue-target congruency and transition interacted such that the decrement in mean accuracy due to cue-target congruency was larger for task repetitions (3.9\%) than for task alternations $(2.1 \%)$ and cue repetitions $(0.9 \%)$.

These observations were supported by a 3 (transition: cue repetition, task repetition, or task alternation) $\times 2$ (cue-target congruency: congruent or incongruent) $\times 5$ (SOA: 0, 100, 200, 400, or $800 \mathrm{~ms}$ ) repeated-measures ANOVA, with the results summarized in Table 1. Nonorthogonal comparisons indicated that mean accuracy decreased from cue repetitions to task repetitions, $F(1$, $54)=4.07, p<.05$, to task alternations, $F(1,54)=5.59, p<.05$. 
Table 1

Summary of Analyses of Variance Conducted on Mean Response Time and Accuracy (as Percentage of Correct Responses) in Experiment 1

\begin{tabular}{|c|c|c|c|c|c|c|c|}
\hline \multirow[b]{2}{*}{ Effect } & \multirow[b]{2}{*}{$d f$} & \multicolumn{3}{|c|}{ Response time } & \multicolumn{3}{|c|}{ Accuracy } \\
\hline & & $F$ & $M S E$ & $\eta^{2}$ & $F$ & $M S E$ & $\eta^{2}$ \\
\hline Transition $(\mathrm{T})$ & 2,54 & $95.76 * *$ & $26,943.08$ & .78 & $9.62 * *$ & 15.74 & .26 \\
\hline Cue-target congruency (C) & 1,27 & $102.43 * *$ & $25,617.98$ & .79 & $18.83 * *$ & 59.19 & .41 \\
\hline Stimulus onset asynchrony (S) & 4, 108 & $293.74 * *$ & $9,315.31$ & .92 & 1.09 & 14.56 & .04 \\
\hline $\mathrm{T} \times \mathrm{C}$ & 2,54 & $19.69 * *$ & $9,233.98$ & .42 & $10.15^{* *}$ & 14.77 & .27 \\
\hline $\mathrm{T} \times \mathrm{S}$ & 8,216 & $3.06^{* *}$ & $8,868.50$ & .10 & 1.39 & 12.43 & .05 \\
\hline $\mathrm{C} \times \mathrm{S}$ & 4, 108 & 1.96 & $8,700.71$ & .07 & 2.24 & 12.35 & .08 \\
\hline $\mathrm{T} \times \mathrm{C} \times \mathrm{S}$ & 8,216 & 1.35 & $10,064.94$ & .05 & 1.86 & 14.61 & .06 \\
\hline
\end{tabular}

Note. $\eta^{2}$ represents the partial $\eta^{2}$ effect size statistic and is unrelated to $\eta$ in the priming model. $* * p<.01$.

Nonorthogonal comparisons indicated that the decrement in mean accuracy due to cue-target congruency was larger for task repetitions than for task alternations, $F(1,54)=7.03, p<.05$, which in turn was marginally larger than for cue repetitions, $F(1,54)=$ $3.35, p=.07$.

Priming model fit. We fit the priming model to the data from Experiment 1 based on the assumptions and equations presented earlier. To produce the transition by cue-target congruency interaction, cue repetitions had separate random walks from task repetitions and task alternations. The random walks for cue repetitions involved setting the evidence for the response category selected on trial $n-1$ for cue $x$ to $\eta_{\mathrm{P}}$, reflecting the temporary cue-response association.

The model fit involved six free parameters: $\eta_{\mathrm{U}}, \eta_{\mathrm{A}}, \eta_{\mathrm{P}}, d, v_{\mathrm{LTM}}$, and $R T_{\text {Base }}$. The values of the parameters were subject to the following constraints: $\eta_{\mathrm{U}} \leq \eta_{\mathrm{A}} \leq \eta_{\mathrm{P}}$ and $0 \leq d \leq 1$. No parameter was allowed to be negative. The response criterion $K$ for compound cue retrieval was fixed at 4.00 based on computer simulations indicating that this value of $K$ resulted in an acceptable level of accuracy. Mean compound cue retrieval time for each type of cue-target congruency was calculated from a set of random walks that represented all possible mappings of response categories onto response keys. Averaging over a set of random walks circumvents the differential bias from targets associated with response categories assigned to the same response key or different response keys.

The priming model was fit to all RT and accuracy data points simultaneously, using the Solver routine in Microsoft Excel to minimize the root-mean-square deviation (RMSD) between the model predictions and the observed data. We report three measures of goodness of fit: RMSD (RT and accuracy), RMSD (RT), and the Pearson product-moment correlation, $r$ (RT). To fit the priming model to RT and accuracy data simultaneously, we multiplied percentage accuracy by 10 to place it on a similar scale to RT (e.g., $98.1 \%$ became 981 ). ${ }^{2}$ The value of RMSD (RT and accuracy) is in milliseconds for RT and in percentages multiplied by 10 for accuracy. For example, RMSD $=20$ would correspond to $20 \mathrm{~ms}$ for RT and $2.0 \%$ for accuracy, although RMSD should be interpreted as a composite measure.

The priming model was fit to the 60 data points ( 30 for RT and 30 for accuracy) given in Appendix A. The values of the best fitting parameters and the RT components from Equation 15 are presented in Table 2. The six-parameter priming model fit the data very well (see Table 2 ), with RMSD (RT and accuracy) $=20$, $\mathrm{RMSD}(\mathrm{RT})=22 \mathrm{~ms}$, and $r(\mathrm{RT})=.989$. The priming model predictions are plotted as lines in the top panel of Figure 1 for the transition data and the top row of Figure 2 for the cue-target congruency data separated by transition.

Consistent with the observed data, the priming model produced differences among transitions based on differential priming of cue encoding. Repetition priming resulted in faster cue encoding for cue repetitions (256 ms) than task repetitions (372 ms), whereas associative priming resulted in faster cue encoding for task repetitions $(372 \mathrm{~ms})$ than task alternations $(453 \mathrm{~ms})$. The differences among transitions decreased with SOA, reflecting the reduction in the contribution of cue encoding to RT. The priming model produced the cue-target congruency effect in both RT and accuracy based on different probabilities of selecting the correct response category from compound cue retrieval (see the sample calculations available in the online supplement at http://dx.doi.org/10.1037/ 0096-3445.134.3.343.supp). The interaction between transition and cue-target congruency was produced from the temporary cue-response associations, with no cue-target congruency effect for cue repetitions preceded by incongruent trials. Note that the same evidence parameters ( $\eta$ values) were capable of modeling the differences in priming of cue encoding and the differences in cue-target congruency. The only noticeable discrepancy was that the priming model predicted the same accuracy for task alternations and task repetitions, but it did predict higher accuracy for cue repetitions. Similar fits to accuracy were obtained in the remaining experiments. The priming model captured all of the critical RT

\footnotetext{
${ }^{2}$ Scaling accuracy by a greater value (e.g., 100) increases RMSD and obscures the fit to RT by exaggerating small differences in accuracy. Scaling accuracy by a lesser value (e.g., 0.1) decreases RMSD and is almost equivalent to not fitting accuracy at all. There is no universally accepted method of scaling accuracy for a model fit to RT and accuracy, but our method has precedent in the literature (e.g., Nosofsky \& Palmeri, 1997). The small range in accuracy across conditions imposes only a weak constraint on the quality of the model fit regardless of the scaling method (which is why we focus on the model fit to the RT data), but it does constrain some of the parameter values in the priming model.
} 
Experiment 1

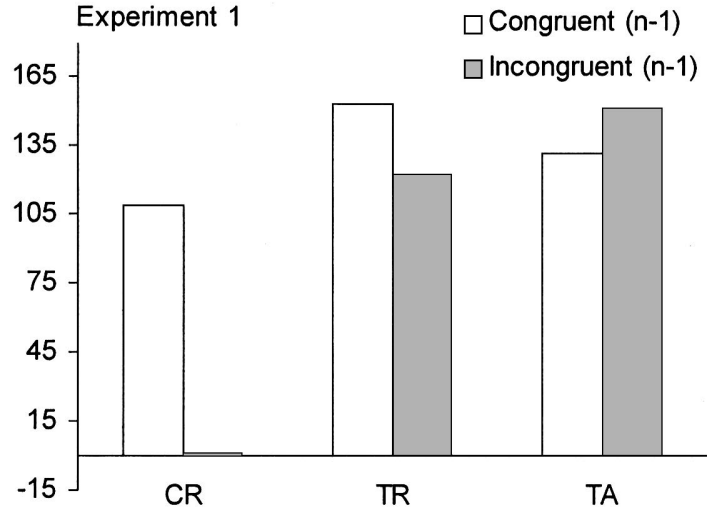

Experiment 2

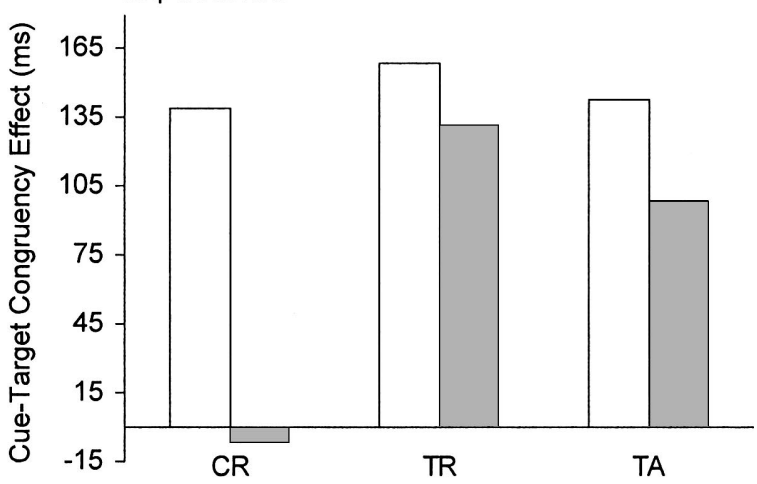

Experiment 3

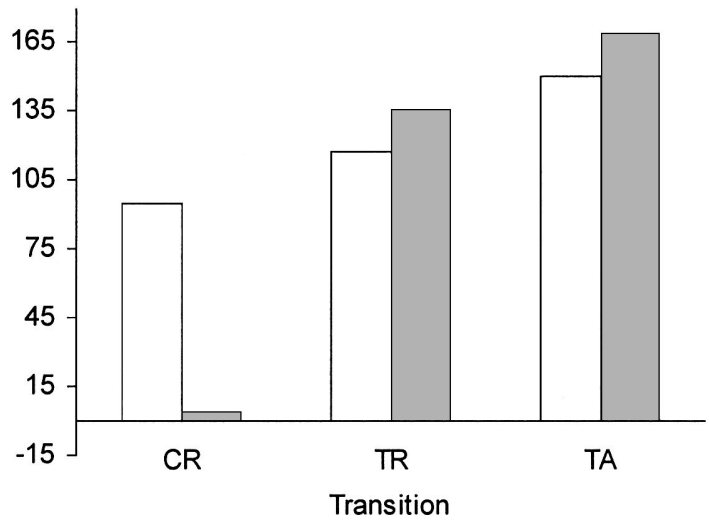

Figure 3. Magnitude of the cue-target congruency effect (the difference in mean response time between incongruent and congruent trials) on trial $n$ as a function of cue-target congruency on trial $n-1$ and transition for Experiments 1, 2, and 3. $\mathrm{CR}=$ cue repetition; $\mathrm{TR}=$ task repetition; $\mathrm{TA}=$ task alternation.

effects and had a good quantitative fit to the data, suggesting that the assumptions and mechanisms composing our priming account are sufficient to explain task-switching performance in the explicit task-cuing procedure.

\section{Experiment 2}

Experiment 1 demonstrated the sufficiency of the priming model. Experiments 2 and 3 replicated and extended the results of
Experiment 1 by contrasting our priming account with the idea that part of task-set reconfiguration involves retrieving task-specific mapping rules (Mayr \& Kliegl, 2000, 2003; also see Rubinstein et al., 2001).

The cues from Experiment 1 are associated with response categories but do not specify the mapping of response categories onto physical response keys. For example, $O D D$ does not

Table 2

Best Fitting Parameter Values ( $R T_{\text {Base }}$ and $R T_{\text {Spatial }}$ in Milliseconds), Calculated Response Time (RT) Components (in Milliseconds), and Measures of the Goodness of Fit of the Priming Model to the RT and Accuracy Data From Experiments 1,2 , and 3

\begin{tabular}{|c|c|c|c|}
\hline \multirow[b]{2}{*}{ Variable } & \multicolumn{3}{|c|}{ Experiment } \\
\hline & 1 & 2 & 3 \\
\hline \multicolumn{4}{|l|}{ Free parameters } \\
\hline $\begin{array}{l}\eta_{\mathrm{U}} \text { (evidence for response categories } \\
\text { unassociated with the presented } \\
\text { cue and target) }\end{array}$ & .0123 & .0172 & .0179 \\
\hline $\begin{array}{l}\eta_{\mathrm{A}} \text { (evidence for response categories } \\
\text { partially associated with the } \\
\text { presented cue })\end{array}$ & .1021 & .1185 & .1222 \\
\hline $\begin{array}{l}\eta_{\mathrm{P}}(\text { evidence for response categories } \\
\text { strongly associated with the } \\
\text { presented cue and target) }\end{array}$ & .3282 & .2209 & .2294 \\
\hline$d$ (short-term memory decay) & .0054 & .0072 & .0050 \\
\hline $\begin{array}{l}\nu_{\mathrm{LTM}}(\text { rate of comparison of the } \\
\text { presented cue to its representation } \\
\text { in long-term memory) }\end{array}$ & .0021 & .0020 & .0019 \\
\hline$R T_{\text {Base }}$ (residual processing time) & 723 & 590 & 516 \\
\hline $\begin{array}{l}R T_{\text {Spatial al }} \text { (time needed to overcome } \\
\text { priming from spatially } \\
\text { incompatible cues) }\end{array}$ & - & - & 24 \\
\hline \multicolumn{4}{|l|}{ Fixed parameter } \\
\hline $\begin{array}{l}K \text { (response criterion for the random } \\
\text { walk) }\end{array}$ & 4.00 & 4.00 & 4.00 \\
\hline \multicolumn{4}{|l|}{ Mean cue encoding times } \\
\hline$\mu$ (for cue repetitions) & 256 & 283 & 332 \\
\hline$\mu$ (for task repetitions) & 372 & 357 & 404 \\
\hline$\mu$ (for task alternations) & 453 & 481 & 513 \\
\hline \multicolumn{4}{|l|}{$\begin{array}{l}\text { Mean compound cue retrieval (random } \\
\text { walk) times }\end{array}$} \\
\hline $\begin{array}{l}R T_{\mathrm{CCR}} \text { (for congruent cue } \\
\text { repetitions) }\end{array}$ & 37 & 83 & 76 \\
\hline $\begin{array}{l}R T_{\mathrm{CCR}} \text { (for congruent task } \\
\quad \text { repetitions and task alternations) }\end{array}$ & 35 & 77 & 71 \\
\hline $\begin{array}{l}R T_{\mathrm{CCR}} \text { (for incongruent cue } \\
\text { repetitions) }\end{array}$ & 106 & 146 & 137 \\
\hline $\begin{array}{l}R T_{\mathrm{CCR}} \text { (for incongruent task } \\
\quad \text { repetitions and task alternations) }\end{array}$ & 173 & 205 & 191 \\
\hline$R T_{\mathrm{CCR}}\left(\right.$ for dual trials) ${ }^{\mathrm{b}}$ & - & 165 & 154 \\
\hline \multicolumn{4}{|l|}{ Measures of goodness of fit } \\
\hline RMSD (RT and accuracy) & 20 & 28 & 30 \\
\hline RMSD $(\mathrm{RT})^{\mathrm{c}}$ & 22 & 30 & 38 \\
\hline$r(\mathrm{RT})^{\mathrm{c}}$ & .989 & .978 & .969 \\
\hline
\end{tabular}

Note. $\quad \mathrm{CCR}=$ compound cue retrieval; $\mathrm{RMSD}=$ root-mean-square deviation between observed data and model predictions; $r=$ productmoment correlation between observed data and model predictions.

${ }^{a}$ This parameter is relevant only for modeling Experiment $3 .{ }^{\mathrm{b}}$ This parameter is relevant only for modeling Experiments 2 and 3. ${ }^{\mathrm{c}}$ These measures are for RT only but come from the model fit to both RT and accuracy. 
indicate whether the left or right response key should be pressed if the correct response category is odd. Mayr and Kliegl (2003) argued that task switching involves retrieving task rules from long-term memory and applying them to the target. Rubinstein et al. (2001) argued that a critical component of task switching involves an executive control process of disabling irrelevant task rules and enabling relevant task rules. Our priming account does not rely on retrieving or enabling task rules to produce switch costs. We acknowledge that the selected response category has to be mapped onto a physical response key, but this process affects the response execution component of $R T_{\text {Base }}$, which is constant across transitions.

If the retrieval of mapping rules is an integral part of switch cost, then embedding the mapping rules within the cue should dramatically alter performance. In Experiment 2, we retained the cues from Experiment 1 (ODD, EVEN, HIGH, and $L O W$ ), but added a new type of cue: the union of the two single-word cues for each task (i.e., ODD-EVEN and HIGH-LOW; see Logan \& Bundesen, 2003, 2004). These dual cues were always spatially compatible with the assigned response-key mappings (i.e., a subject with $O D D-E V E N$ and $H I G H-L O W$ as dual cues would press the left key for odd and high and the right key for even and low). Dual cues represent both of the relevant response categories on each trial, so they are both congruent and incongruent with the target, resulting in dual cue-target congruency. The single-word cues are strongly associated with only one of the response categories, making them congruent or incongruent with the target.

If task-switching performance is linked to the retrieval of mapping rules, then RT should be much faster for dual cues than congruent or incongruent single-word cues because the mapping rules are explicitly indicated. If switch costs are associated with the retrieval of mapping rules, then one would also expect switch costs to be smaller with dual cues. In contrast, our priming account predicts an intermediate RT for dual cues with respect to congruent and incongruent singleword cues because they would provide equal evidence for each response category in compound cue retrieval. Switch costs for dual cues should be similar to those for single-word cues because cue encoding does not involve the retrieval of mapping rules. The only change that might be expected in the priming model fit is a smaller $R T_{\text {Base }}$, reflecting facilitation on some trials in mapping the selected response category onto a physical response key.

\section{Method}

Experiment 2 was conducted according to the procedure described in the General Method section, but with the following modifications. Two dual cues representing unions of the single-word task-specific cues were added, with the restriction that the order of the constituent words be spatially compatible with the subject's response-key mappings. For example, the dual cues for a subject with the responses odd and high mapped onto the $C$ key and the responses even and low mapped onto the $M$ key would be ODD-EVEN and HIGH-LOW. A subject with the reversed response-key mappings for both tasks would have EVEN-ODD and $L O W-H I G H$ as dual cues. Two minor changes were made to the procedure: The examples of incongruent cue-target combinations were included in the on screen instructions, and subjects were reminded of the response-key mappings during the rest periods.

\section{Results and Discussion}

The data were classified according to the procedure described in the General Method section, with dual cues classified as having dual cue-target congruency. Mean RT and accuracy (as percentage of correct responses) were calculated for each subject for all combinations of transition, cue-target congruency, and SOA; these data are presented in Appendix B. Accuracy was high and within-subject correlations between RT and accuracy did not indicate any speed-accuracy trade-offs; therefore, the analyses focused on RT.

RT analysis. Mean RTs across subjects for each transition as a function of SOA are presented in the middle panel of Figure 1, and the partitioning of this data by cue-target congruency is presented in the middle row of Figure 2. The pattern of results replicates Experiment 1: Mean RT for cue repetitions (853 ms) was faster than for task repetitions ( $943 \mathrm{~ms}$ ), which in turn was faster than for task alternations $(1,037 \mathrm{~ms})$. The difference between task alternations and task repetitions did not differ among incongruent, congruent, and dual trials (95, 102, and 87 ms, respectively), consistent with our priming account but inconsistent with mapping rule retrieval accounts (e.g., Mayr \& Kliegl, 2003; Rubinstein et al., 2001). Mean RT and the differences among transitions generally decreased as SOA increased, although there was some instability in the time-course functions (see Figure 1). There was a cue-target congruency effectmean RT for incongruent trials (996 ms) was slower than for congruent trials (882 ms)-but cue-target congruency interacted with transition (see Figure 2): The cue-target congruency effect was smaller for cue repetitions $(73 \mathrm{~ms})$ than task repetitions $(139 \mathrm{~ms})$ and task alternations $(132 \mathrm{~ms})$, which did not differ. Dual trials had an intermediate mean RT (955 ms) as predicted by our priming account.

These observations were supported by a 3 (transition: cue repetition, task repetition, or task alternation) $\times 3$ (cue-target congruency: congruent, incongruent, or dual) $\times 5$ (SOA: 0, $100,200,400$, or $800 \mathrm{~ms}$ ) repeated-measures ANOVA, with the results summarized in Table 3. Nonorthogonal comparisons indicated that mean RT for cue repetitions was faster than for task repetitions, $F(1,54)=74.32, p<.01$, which in turn was faster than for task alternations, $F(1,54)=83.45, p<.01$. Nonorthogonal comparisons indicated that mean RT for incongruent trials was slower than for dual trials, $F(1,54)=12.01$, $p<.01$, which in turn was slower than for congruent trials, $F(1$, $54)=37.20, p<.01$. Orthogonal comparisons revealed that the cue-target congruency effect was smaller for cue repetitions than for the mean of task repetitions and task alternations, $F(1$, $108)=16.15, p<.01$, which did not differ, $F(1,108)<1$. Additional comparisons indicated that the difference between task alternations and task repetitions did not differ by cuetarget congruency, $F \mathrm{~s}(1,108)<1$.

As in Experiment 1, mean RTs were calculated for each subject based on the transition and cue-target congruency on trial $n$ with respect to the cue-target congruency on trial $n-1$; these data are depicted as cue-target congruency effects for trial $n$ in the middle 
Table 3

Summary of Analyses of Variance Conducted on Mean Response Time and Accuracy (as Percentage of Correct Responses) in Experiment 2

\begin{tabular}{|c|c|c|c|c|c|c|c|}
\hline \multirow[b]{2}{*}{ Effect } & \multirow[b]{2}{*}{$d f$} & \multicolumn{3}{|c|}{ Response time } & \multicolumn{3}{|c|}{ Accuracy } \\
\hline & & $F$ & $M S E$ & $\eta^{2}$ & $F$ & $M S E$ & $\eta^{2}$ \\
\hline Transition $(\mathrm{T})$ & 2,54 & $157.68 * *$ & $22,587.63$ & .85 & $53.32 * *$ & 27.13 & .66 \\
\hline Cue-target congruency (C) & 2,54 & $46.90 * *$ & $29,990.30$ & .64 & $20.79 * *$ & 48.38 & .44 \\
\hline Stimulus onset asynchrony (S) & 4,108 & $290.11 * *$ & $13,888.72$ & .92 & 1.32 & 21.33 & .05 \\
\hline $\mathrm{T} \times \mathrm{C}$ & 4,108 & $5.10 * *$ & $11,323.44$ & .16 & $3.96^{* *}$ & 20.34 & .13 \\
\hline $\mathrm{T} \times \mathrm{S}$ & 8,216 & $4.22 * *$ & $10,274.57$ & .14 & 1.03 & 20.55 & .04 \\
\hline $\mathrm{C} \times \mathrm{S}$ & 8,216 & $2.32 *$ & $11,870.25$ & .08 & $4.01 * *$ & 20.03 & .13 \\
\hline $\mathrm{T} \times \mathrm{C} \times \mathrm{S}$ & 16,432 & 1.47 & $10,872.65$ & .05 & 0.80 & 17.77 & .03 \\
\hline
\end{tabular}

Note. $\eta^{2}$ represents the partial $\eta^{2}$ effect size statistic and is unrelated to $\eta$ in the priming model. $* p<.05$. ** $p<.01$.

panel of Figure $3{ }^{3}$ There was a three-way interaction by which the cue-target congruency effect was eliminated (if not slightly reversed) when the preceding trial was incongruent for cue repetitions.

These observations were supported by a 3 (transition: cue repetition, task repetition, or task alternation) $\times 2$ (cue-target congruency on trial $n$ : incongruent or congruent) $\times 2$ (cue-target congruency on trial $n-1$ : incongruent or congruent) repeatedmeasures ANOVA, collapsing across SOA and excluding the first two trials of each block. To avoid redundancy, we report only statistics associated with cue-target congruency on trial $n-1$. There was a main effect of cue-target congruency on trial $n-1$, $F(1,27)=24.39, M S E=4,539.81, p<.01, \eta^{2}=.48$, and it interacted with transition, $F(2,54)=7.54, M S E=6,556.95, p<$ $.01, \eta^{2}=.22$, and cue-target congruency on trial $n, F(1,27)=$ 19.77, $M S E=5,556.71, p<.01, \eta^{2}=.42$. The three-way interaction was almost significant, $F(2,54)=3.12, M S E=$ 9,163.75, $p=.052, \eta^{2}=.10$. A three-way interaction contrast comparing the differences in cue-target congruency on trial $n$ by the cue-target congruency on trial $n-1$ for cue repetitions to the mean of those differences for task repetitions and task alternations was significant, $F(1,54)=15.25, p<.01$.

Accuracy analysis. Overall accuracy across subjects was high ( $M=95.5 \%$; see Appendix B). Mean accuracy decreased from cue repetitions $(97.5 \%)$ to task repetitions $(96.7 \%)$ to task alternations (94.0\%). Mean accuracy was lower for incongruent trials $(94.3 \%)$ than congruent and dual trials (both $97.0 \%$ ). Cue-target congruency and transition interacted such that the decrement in mean accuracy due to cue-target congruency was smaller for cue repetitions $(1.1 \%)$ than task repetitions $(3.3 \%)$ and task alternations (3.6\%), which did not differ.

These observations were supported by a 3 (transition: cue repetition, task repetition, or task alternation) $\times 3$ (cue-target congruency: congruent, incongruent, or dual) $\times 5$ (SOA: 0, 100, 200, 400 , or $800 \mathrm{~ms}$ ) repeated-measures ANOVA, with the results summarized in Table 3. Nonorthogonal comparisons indicated that mean accuracy decreased from cue repetitions to task repetitions, $F(1,54)=5.57, p<.05$, to task alternations, $F(1,54)=56.67$, $p<.01$. Orthogonal comparisons indicated lower mean accuracy for incongruent trials compared with the mean of congruent and dual trials, $F(1,54)=41.59, p<.01$, which did not differ, $F(1$, $54)<1$. Orthogonal comparisons indicated that the decrement in mean accuracy due to cue-target congruency was smaller for cue repetitions than for the mean of task repetitions and task alternations, $F(1,108)=13.68, p<.01$, which did not differ, $F(1$, $108)<1$.

Priming model fit. We fit the priming model to the data from Experiment 2 using the same method as in Experiment 1. To calculate the time of the random walk for dual cues, we assumed that dual cues do not provide the strongest evidence $\left(\eta_{\mathrm{P}}\right)$ to each response category linked to their constituent cues but function as cues that are associated with their constituent cues, such that an intermediate amount of evidence $\left(\eta_{\mathrm{A}}\right)$ is given to each associated response category. The rationale for this implementation was that a dual cue (assumed to be a unitary percept) represents only a partial perceptual match to its constituent cues but is associated conceptually with both of the relevant response categories. Consequently, both response categories associated with a dual cue were given evidence of equal strength in the random walk. Dual cues were also assumed to function as associated cues for task repetitions.

The priming model was fit to the 90 data points ( 45 for RT and 45 for accuracy) given in Appendix B. The values of the best fitting parameters and the RT components from Equation 15 are presented in Table 2. Although there were 30 more data points than in Experiment 1, the six-parameter priming model fit the data well (see Table 2), with RMSD (RT and accuracy) = 28, RMSD $(\mathrm{RT})=30 \mathrm{~ms}$, and $r(\mathrm{RT})=.978$. The priming model predictions are plotted as lines in the middle panel of Figure 1 for the transition data and the middle row of Figure 2 for the cue-target congruency data separated by transition.

\footnotetext{
${ }^{3}$ In this analysis, dual cues were omitted because their relationship to the preceding trial differs from single-word cues depending on the transition. For cue repetitions, single-word cues on trial $n$ can be preceded by incongruent or congruent cues on trial $n-1$; dual cues can only be preceded by dual cues. For task repetitions, single-word cues on trial $n$ can be preceded by incongruent, congruent, or dual cues on trial $n-1$; dual cues can only be preceded by incongruent or congruent single-word cues. Our primary interest in conducting this analysis was to assess how the cue-target congruency effect (the difference between incongruent and congruent trials) differs based on the cue-target congruency of trial $n-1$, so dual cues were not considered to be very informative.
} 
As in Experiment 1, the priming model produced the differences among transitions, the reduction in these differences with SOA, the cue-target congruency effect, and the interaction between transition and cue-target congruency. Aside from the priming models predicting the same accuracy for task alternations and task repetitions, the only noticeable discrepancy was that RTs for incongruent and dual trials for cue repetitions were slightly reversed in the model fit with respect to the experimental data. This discrepancy could be due to the insensitivity of the priming model to fluctuations in dual cue-target congruency across SOA (see Figure 2 ) or the need to modify our assumption about the amount of evidence provided by dual cues (i.e., $\eta_{\mathrm{A}}$ may be too low). The priming model captured most of the RT effects and had a good quantitative fit to the data, suggesting that retrieval of mapping rules is not an integral part of "switch cost." Priming of cue encoding and compound cue retrieval appear to be sufficient to explain task-switching performance in the explicit task-cuing procedure.

\section{Experiment 3}

Experiment 2 provided converging evidence for our priming account through the modeling of dual cues. Experiment 3 replicates and extends the results of Experiments 1 and 2 by examining a stronger manipulation concerning the availability of mapping rules.

The constituent words of the dual cues in Experiment 2 were always spatially compatible with the subject's response-key mappings. Our priming account predicts that dual cues could provide evidence for their associated response categories regardless of the order of their constituent words but that order might affect the mapping of the selected response category onto a physical response key. In Experiment 3, we allowed single-word cues and dual cues to be either spatially compatible or incompatible on each trial. If switch costs are associated with the retrieval of mapping rules, then spatially incompatible cues should substantially impair task-switching performance, producing large switch costs and low accuracy. In contrast, our priming account predicts little effect of spatial compatibility, except for slower response execution with spatially incompatible cues, consistent with extant research on stimulus-response compatibility (e.g., Hommel \& Prinz, 1997). We expect a small spatial compatibility effect, with slower RTs for spatially incompatible cues due to the need to overcome priming of the spatial location of the incorrect physical response key. The differences among transitions and the cue-target congruency effects should remain similar to Experiment 2 according to our priming account. Any interaction between cue-target congruency and spatial compatibility would likely involve modulation of the spatial compatibility effect, not cue-target congruency.

\section{Method}

Experiment 3 was conducted according to the procedure described in the General Method section, with the following modifications. The cues from Experiment 2 were retained, but their spatial compatibility was manipulated. All cues included a hyphen that served as a central point of spatial reference. Single-word cues could appear to the left or right of the hyphen (e.g., $O D D$ - and - $O D D$ ). The constituents of dual cues could also appear on either side of the hyphen (e.g., $O D D-E V E N$ and $E V E N-O D D$ ). Single-word and dual cues could be spatially compatible or incompatible with the response-key mapping assigned to the subject. For example, a subject with the responses odd and high mapped onto the $C$ key and the responses even and low mapped onto the $M$ key would have $O D D-, H I G H-,-E V E N,-L O W$, $O D D-E V E N$, and $H I G H-L O W$ as spatially compatible cues, with a corresponding reversed set of spatially incompatible cues. The target on each trial was centered with respect to the hyphen, making it spatially neutral.

\section{Results and Discussion}

Mean RT and accuracy (as percentage of correct responses) were calculated for each subject for all combinations of transition, cue-target congruency, spatial compatibility, and SOA; these data are presented in Appendix C. ${ }^{4}$ Accuracy was high and withinsubject correlations between RT and accuracy did not indicate any speed-accuracy trade-offs; therefore, the analyses focused on RT.

$R T$ analysis. Mean RTs across subjects for each transition as a function of SOA are presented in the bottom panel of Figure 1, and the partitioning of these data by cue-target congruency is presented in the bottom row of Figure 2. The pattern of results replicates Experiments 1 and 2: Mean RT for cue repetitions (821 $\mathrm{ms}$ ) was faster than for task repetitions $(910 \mathrm{~ms})$, which in turn was faster than for task alternations (991 ms). The difference between task alternations and task repetitions did not differ among incongruent, congruent, and dual trials $(87,68$, and $87 \mathrm{~ms}$, respectively). Mean RT and the differences among transitions generally decreased as SOA increased, although there was some instability in the time-course functions (see Figure 1). There was a cue-target congruency effect-mean RT for incongruent trials (961 ms) was slower than for congruent trials $(852 \mathrm{~ms})$, with dual trials at an intermediate mean RT (909 ms) - but cue-target congruency interacted with transition: The cue-target congruency effect was smaller for cue repetitions (46 ms) than task repetitions (130 ms) and task alternations (149 ms), which did not differ.

The critical factor in Experiment 3 was spatial compatibility, for which there was only a main effect: Mean RT for incompatible trials (919 ms) was slower than for compatible trials (895 ms). The magnitude of the effect and the lack of any interactions with transition or cue-target congruency provide strong evidence that retrieval of mapping rules is not a critical determinant of switch costs. The spatial compatibility effect is consistent with our priming account and the idea that response execution is slowed by spatially incompatible cues.

These observations were supported by a 3 (transition: cue repetition, task repetition, or task alternation) $\times 3$ (cue-target con-

\footnotetext{
${ }^{4}$ The addition of the spatial compatibility factor and no constraints on the random selection of cues and targets resulted in very rare circumstances in which some subjects did not have any trials contributing to specific cell means. The missing data affected only 20 of the 2,520 cell means and was evenly distributed across subjects. To permit an ANOVA of the full experimental design, we estimated the missing cell means with single imputation based on a subject's marginal mean for the transition, the condition cell mean computed across subjects with data, and the grand mean. Given that less than $1 \%$ of the data had to be estimated, we do not believe our results or the conclusions drawn from them are compromised. To assess the influence of the estimated data, we computed separate ANOVAs for complete data sets collapsed across either SOA or spatial compatibility and compared them to the ANOVA for the full design using the estimated data. The patterns of statistical significance for the common effects were very similar, suggesting that our estimation method did not generate any serious anomalies in the data.
} 
gruency: congruent, incongruent, or dual) $\times 2$ (spatial compatibility: incompatible or compatible) $\times 5$ (SOA: $0,100,200,400$, or $800 \mathrm{~ms}$ ) repeated-measures ANOVA, with the results summarized in Table 4. Nonorthogonal comparisons indicated that mean RT for cue repetitions was faster than for task repetitions, $F(1,54)=$ $45.38, p<.01$, which in turn was faster than for task alternations, $F(1,54)=36.70, p<.01$. Nonorthogonal comparisons indicated that mean RT for incongruent trials was slower than for dual trials, $F(1,54)=37.95, p<.01$, which in turn was slower than for congruent trials, $F(1,54)=46.45, p<.01$. Orthogonal comparisons revealed that the cue-target congruency effect was smaller for cue repetitions than for the mean of task repetitions and task alternations, $F(1,108)=40.01, p<.01$, which did not differ, $F(1$, $108)=1.21, p=.27$. Additional comparisons indicated that the difference between task alternations and task repetitions did not differ by cue-target congruency, $F \mathrm{~s}(1,108)<1.66, p \mathrm{~s}>.20$.

As in Experiments 1 and 2, mean RTs were calculated for each subject based on the transition and cue-target congruency on trial $n$ with respect to the cue-target congruency on trial $n-1$; these data are depicted as cue-target congruency effects for trial $n$ in the bottom panel of Figure 3 (see Footnote 3). There was a three-way interaction by which the cue-target congruency effect was markedly reduced when the preceding trial was incongruent for cue repetitions, replicating previous results.

These observations were supported by a 3 (transition: cue repetition, task repetition, or task alternation) $\times 2$ (cue-target congruency on trial $n$ : incongruent or congruent) $\times 2$ (cue-target congruency on trial $n-1$ : incongruent or congruent) repeatedmeasures ANOVA, collapsing across spatial compatibility and SOA and excluding the first two trials of each block. To avoid redundancy, we report only statistics associated with cue-target congruency on trial $n-1$. There was a main effect of cue-target congruency on trial $n-1, F(1,27)=27.16, M S E=7,120.80, p<$ $.01, \eta^{2}=.50$, but in contrast to Experiments 1 and 2, it did not participate in significant two-way interactions with transition, $F(2$,
$54)=2.12, M S E=9,283.71, p=.13, \eta^{2}=.07$, or cue-target congruency on trial $n, F(1,27)<1$. The three-way interaction was significant, $F(2,54)=3.74, M S E=7,577.13, p<.05, \eta^{2}=.12$. A three-way interaction contrast comparing the differences in cue-target congruency on trial $n$ by the cue-target congruency on trial $n-1$ for cue repetitions to the mean of those differences for task repetitions and task alternations was significant, $F(1,54)=$ 15.37, $p<.01$.

Accuracy analysis. Overall accuracy across subjects was high ( $M=96.1 \%$; see Appendix C). Mean accuracy decreased from cue repetitions $(97.2 \%)$ to task repetitions $(96.5 \%)$ to task alternations (95.3\%). Mean accuracy was lower for incongruent trials $(94.0 \%)$ than congruent trials $(97.9 \%)$ and dual trials $(97.1 \%)$, which did not differ. Cue-target congruency and transition interacted such that the decrement in mean accuracy due to cue-target congruency was smaller for cue repetitions $(2.0 \%)$ than for task repetitions $(5.0 \%)$ and task alternations $(4.6 \%)$, which did not differ. Mean accuracy was the same for spatially incompatible and compatible trials $(96.2 \%$ and $96.5 \%$, respectively), consistent with our priming account because the probability of selecting the correct response by compound cue retrieval is unrelated to spatial compatibility.

These observations were supported by a 3 (transition: cue repetition, task repetition, or task alternation) $\times 3$ (cue-target congruency: congruent, incongruent, or dual) $\times 2$ (spatial compatibility: incompatible or compatible) $\times 5$ (SOA: $0,100,200,400$, or $800 \mathrm{~ms}$ ) repeated-measures ANOVA, with the results summarized in Table 4. Nonorthogonal comparisons indicated that mean accuracy decreased from cue repetitions to task repetitions, $F(1,54)=$ 5.08, $p<.05$, to task alternations, $F(1,54)=11.35, p<.01$. Orthogonal comparisons indicated lower mean accuracy for incongruent trials compared with the mean of congruent and dual trials, $F(1,54)=68.32, p<.01$, which did not differ, $F(1,54)=3.25$, $p=.08$. Orthogonal comparisons indicated that the decrement in mean accuracy due to cue-target congruency was smaller for cue repetitions than for the mean of task repetitions and task alterna-

Table 4

Summary of Analyses of Variance Conducted on Mean Response Time and Accuracy (as Percentage of Correct Responses) in Experiment 3

\begin{tabular}{|c|c|c|c|c|c|c|c|}
\hline \multirow[b]{2}{*}{ Effect } & \multirow[b]{2}{*}{$d f$} & \multicolumn{3}{|c|}{ Response time } & \multicolumn{3}{|c|}{ Accuracy } \\
\hline & & $F$ & MSE & $\eta^{2}$ & $F$ & $M S E$ & $\eta^{2}$ \\
\hline Transition $(\mathrm{T})$ & 2,54 & $81.94 * *$ & $74,718.57$ & .75 & $16.01 * *$ & 46.18 & .37 \\
\hline Cue-target congruency (C) & 2,54 & $84.25 * *$ & $29,272.87$ & .76 & $35.79 * *$ & 98.81 & .57 \\
\hline Spatial compatibility $(\mathrm{P})$ & 1,27 & 12.70 ** & $28,708.47$ & .32 & 1.08 & 54.69 & .04 \\
\hline Stimulus onset asynchrony (S) & 4,108 & $372.00 * *$ & $25,752.16$ & .93 & 1.89 & 44.59 & .07 \\
\hline $\mathrm{T} \times \mathrm{C}$ & 4,108 & $11.57 * *$ & $20,416.47$ & .30 & $5.01 * *$ & 53.24 & .16 \\
\hline $\mathrm{T} \times \mathrm{P}$ & 2,54 & 0.84 & $23,815.07$ & .03 & 0.32 & 41.12 & .01 \\
\hline $\mathrm{T} \times \mathrm{S}$ & 8,216 & $3.67 * *$ & $27,014.31$ & .12 & 0.60 & 62.94 & .02 \\
\hline $\mathrm{C} \times \mathrm{P}$ & 2,54 & 2.28 & $24,636.06$ & .08 & 0.15 & 42.30 & .01 \\
\hline $\mathrm{C} \times \mathrm{S}$ & 8,216 & $3.67 * *$ & $22,904.33$ & .12 & $2.18 *$ & 44.36 & .08 \\
\hline $\mathrm{P} \times \mathrm{S}$ & 4,108 & 0.27 & $21,092.96$ & .01 & 1.51 & 52.11 & .05 \\
\hline $\mathrm{T} \times \mathrm{C} \times \mathrm{P}$ & 4,108 & 0.09 & $23,568.92$ & .00 & 0.91 & 39.97 & .03 \\
\hline $\mathrm{T} \times \mathrm{C} \times \mathrm{S}$ & 16,432 & 0.46 & $23,593.74$ & .02 & 0.49 & 52.15 & .02 \\
\hline $\mathrm{T} \times \mathrm{P} \times \mathrm{S}$ & 8,216 & 1.11 & $20,471.34$ & .04 & 1.85 & 52.05 & .06 \\
\hline $\mathrm{C} \times \mathrm{P} \times \mathrm{S}$ & 8,216 & 0.17 & $25,231.89$ & .01 & 0.96 & 53.75 & .03 \\
\hline $\mathrm{T} \times \mathrm{C} \times \mathrm{P} \times \mathrm{S}$ & 16,432 & 1.21 & $26,210.60$ & .04 & 0.74 & 52.20 & .03 \\
\hline
\end{tabular}

Note. $\eta^{2}$ represents the partial $\eta^{2}$ effect size statistic and is unrelated to $\eta$ in the priming model.

$* p<.05$. ** $p<.01$. 
tions, $F(1,108)=13.93, p<.01$, which did not differ, $F(1$, $108)<1$.

Priming model fit. We fit the priming model to the data from Experiment 3 using the same method as in Experiments 1 and 2. We assumed that spatially incompatible cues prime the spatial location of the incorrect response key. Such priming would slow the mapping of the selected response category onto a physical response key, increasing $R T_{\text {Base }}$. We modeled spatial compatibility by adding a small RT component, $R T_{\text {Spatial }}$, to all spatially incompatible trials.

The priming model was fit to the 180 data points ( 90 for RT and 90 for accuracy) given in Appendix C. The values of the best fitting parameters and the RT components from Equation 15 are presented in Table 2. Although there were 90 more data points than in Experiment 2 and 120 more data points than in Experiment 1, the seven-parameter priming model fit the data well (see Table 2), with RMSD (RT and accuracy) $=30, \operatorname{RMSD}(\mathrm{RT})=38 \mathrm{~ms}$, and $r(\mathrm{RT})=.969$. The priming model predictions are plotted as lines in the bottom panel of Figure 1 for the transition data and the bottom row of Figure 2 for the cue-target congruency data separated by transition, collapsed across spatial compatibility to highlight the similarity to Experiment 2.

As in Experiments 1 and 2, the priming model produced the differences among transitions, the reduction in these differences with SOA, the cue-target congruency effect, and the interaction between transition and cue-target congruency. Consistent with the model fit in Experiment 2, RTs for incongruent and dual trials for cue repetitions were slightly reversed with respect to the experimental data. The relative values of the best fitting parameters for Experiment 3 were very similar to those for Experiment 2. For example, the ratios of $\eta_{\mathrm{U}}$ to $\eta_{\mathrm{P}}$ and $\eta_{\mathrm{A}}$ to $\eta_{\mathrm{P}}$ were .078 and .536 for Experiment 2 and .078 and .533 for Experiment 3. The priming model captured the RT effects observed in the previous experiments and also produced a spatial compatibility effect of $24 \mathrm{~ms}$ with the $R T_{\text {Spatial }}$ parameter, which is identical in magnitude to the observed spatial compatibility effect. These results are inconsistent with the idea that "switch cost" depends on the retrieval of mapping rules, but they are consistent with our priming account, in which spatial compatibility does not affect cue encoding or compound cue retrieval.

\section{General Discussion}

A short-term priming account of explicitly cued performance in task-switching situations was developed and assessed in three experiments. The data were highly consistent across experiments, with repeated cue encoding benefits, switch costs, and cue-target congruency effects. All of these results-including the "switch costs" and their reduction with preparation time-could be reproduced by a set of basic psychological processes that were instantiated in a mathematical model. ${ }^{5}$ These processes include priming of cue encoding from residual activation in STM and compound cue retrieval of response categories from long-term memory, but no executive control process such as task-set reconfiguration.

Our priming account advances our understanding of the processes involved in the explicit task-cuing procedure. The priming model formalizes the compound stimulus strategy suggested by Logan and Bundesen $(2003,2004)$ and represents an instantiation of the compound cue retrieval theory proposed by Ratcliff and
McKoon (1988). Previous accounts of performance involving a compound stimulus strategy (Arrington \& Logan, 2004; Logan \& Bundesen, 2003, 2004) were based only on abstract descriptions of how such a strategy might be enacted (e.g., "the cue and the target act as a compound stimulus that uniquely determines the correct response," Logan \& Bundesen, 2003, pp. 577-578). By formalizing compound cue retrieval, we illustrated how responses could be selected based on combined evidence from cues and targets participating in a random walk process (Dosher \& Rosedale, 1989; Ratcliff \& McKoon, 1988).

Previous accounts of the differences among transitions did not provide mechanisms for generating such differences. For example, although Logan and Bundesen (2003) presented Equation 4 in their account of explicitly cued performance, mean cue encoding times were free parameters constrained by data. The priming model involves calculation of mean cue encoding times based on rates of comparison of the cue to representations in short- and long-term memory, with differential residual activation influencing the rate of comparison to STM to produce the differences among transitions. Past attempts to model switch costs were restricted to adding a small amount of task-switching time (as in Model $2+1$ of Logan \& Bundesen, 2003) or changing $R T_{\text {Base }}$ to reflect proposed differences in target processing time (as in Model 3 of Logan \& Bundesen, 2004; also see Arrington \& Logan, 2004). "Switch costs" can arise naturally in the priming model from differences in priming of cue encoding. In this regard, the priming model offers an advantage over models that never predict differences between task repetitions and task alternations (e.g., Model 2 of Logan \& Bundesen, 2003).

Considering its success in explaining task-switching performance, we believe our priming account represents another step toward removing the enigma surrounding the role of executive control in task switching (cf. Monsell \& Driver, 2000). The priming model clearly demonstrates how a set of basic psychological processes can produce performance that appears to reflect the action of executive control processes.

\section{Applications}

Our priming account is not restricted to the experiments presented in this article. The priming model should be capable of explaining differences among transitions observed in past studies involving the explicit task-cuing procedure, at least with meaningful word cues. The present experiments involved strongly associated cues, but we demonstrate the flexibility of the priming model by fitting it to experiments involving weakly associated cues.

Logan and Bundesen (2003) investigated differences among transitions using two cues per task. In their Experiment 3, they observed a large repeated cue encoding benefit of $168 \mathrm{~ms}$ and a small switch cost of 35 ms. Model $2+1$ (which includes a repeated cue encoding benefit and task-switching time) fit well, with RMSD $=34 \mathrm{~ms}$ and $r=.972$, which was slightly but not significantly better than Model 2 (which includes only a repeated

\footnotetext{
5 The mathematical model results were corroborated by computer simulations programmed in $\mathrm{C}$ that implemented the assumptions and mechanisms in our priming account. The correlation between the model and simulation predictions exceeded .999 for all experiments.
} 
cue encoding benefit), with RMSD $=35 \mathrm{~ms}$ and $r=.971$. The cues used for each task in this experiment could be considered only weakly associated for at least two reasons. First, ODD-EVEN and HIGH-LOW specify response categories, whereas PARITY and MAGNITUDE are more abstract task labels. Second, there is little evidence from free association norms that the cues are strongly associated: The highest production proportion was for $M A G$ NITUDE-HIGH (.049) and PARITY was never produced in response to $O D D$ or $E V E N$ (Nelson et al., 1999). On the basis of these weak associations, our priming account would predict little or no switch cost, consistent with the data.

The priming model was fit to the $30 \mathrm{RT}$ data points in this experiment (see Logan \& Bundesen, 2003), with the constraint that compound cue retrieval obtains overall accuracy of $95 \%$, as observed in the data. Residual processing time was subsumed in the random walk (removing $R T_{\text {Base }}$ as a parameter), whereas the reverse was done by Logan and Bundesen (2003). The fiveparameter priming model fit well, with RMSD $=32 \mathrm{~ms}$ and $r=$ .975 , producing a small "switch cost" of $32 \mathrm{~ms}$.

In their Experiment 4, which involved different tasks and cues, Logan and Bundesen (2003) observed a repeated cue encoding benefit of $95 \mathrm{~ms}$ and a switch cost of $14 \mathrm{~ms}$. Models 2 and $2+1 \mathrm{fit}$ equally well, with RMSD $=11 \mathrm{~ms}$ and $r=.995$ for Model 2, and $\mathrm{RMSD}=10 \mathrm{~ms}$ and $r=.996$ for Model $2+1$. The priming model was fit to the $30 \mathrm{RT}$ data points in this experiment, with the constraint that compound cue retrieval obtains overall accuracy of $96 \%$, as observed in the data. The five-parameter priming model fit as well as Model $2+1$, with RMSD $=10 \mathrm{~ms}$ and $r=.996$, plus a small "switch cost" of $14 \mathrm{~ms}$.

These fits of the priming model to Experiments 3 and 4 of Logan and Bundesen (2003) demonstrate its flexibility in accounting for other data, especially considering the fact that it did as well as (if not slightly better than) a model that included time for task switching. The goodness of fit of the priming model could be attributed partly to one or two extra parameters (Model $2+1$ had four parameters and Models 1 and 2 each had three parameters), but the priming model is specified in greater detail and is the only model that can be fit to both RT and accuracy. Note that the small "switch costs" in the fits to Logan and Bundesen (2003) are consistent with our priming account: Weak associations between cues would produce weak associative priming for task repetitions. Larger "switch costs" were observed in the present experiments because of stronger associative priming based on stronger associations between cues.

\section{Limitations and Extensions}

Our main objective in developing the priming model was not to account for all task-switching phenomena in all task-switching procedures. We focused on "switch costs" and their reduction with SOA in the explicit task-cuing procedure because those effects have been interpreted as strong evidence for executive control processes such as task-set reconfiguration. Although the priming model can account for such effects without task-set reconfiguration, we do not argue that it provides a complete account of explicitly cued performance nor performance in task-switching situations in which explicit cues are absent. In this section, we consider some limitations of the present formulation of the priming model and possible extensions that might allow it to account for a wider range of phenomena.

Long-term priming effects. Priming of cue encoding in the priming model is based solely on the immediately preceding trial, which is why we refer to it as a short-term priming account. Many researchers have found evidence for long-term priming of performance in task-switching situations (e.g., Allport \& Wylie, 1999, 2000; Koch \& Allport, in press; Waszak et al., 2003, 2004; Wylie $\&$ Allport, 2000). We believe the priming model can be extended to include both short- and long-term priming to provide a more comprehensive account of task-switching data.

Graded residual activation of cues from multiple preceding trials could prime cue encoding on each trial, with the immediately preceding trial contributing the greatest amount of residual activation (cf. Altmann, 2002). If cues are stored as individual traces in long-term memory, the evidence for the presented cue could be expressed as the sum of power-function decayed traces of the cue (e.g., Anderson \& Matessa, 1997):

$$
\eta(i \mid x)=\sum_{m=1}^{M} \eta^{\prime}\left(i_{m} \mid x\right) \cdot t_{m}^{-d},
$$

where $\eta^{\prime}\left(i_{m} \mid x\right)$ is the evidence for the $m$ th instance of a cue that occurred $t_{m}$ ms ago and $d$ is the decay constant.

Combinations of cues, targets, and responses could also be included in extensions to the priming model. Whether they are interpreted as instances (Logan, 1988) or other types of stimulusresponse bindings (Allport \& Wylie, 2000; Koch \& Allport, in press; Koch, Prinz, \& Allport, 2005), these combinations could accumulate in long-term memory and be retrieved on a given trial to positively or negatively prime cue encoding, target encoding, response selection, or residual processes. For example, by manipulating transition frequency, we obtained evidence for faster cue encoding when a transition is frequent (Schneider \& Logan, in press). One interpretation of this finding is that cue encoding might be primed by retrieval of accumulated instances of past transitions. Priming of processes that follow cue encoding could alter compound cue retrieval or shift $R T_{\text {Base }}$ (as spatial compatibility did in Experiment 3) and produce so-called residual switch costs. Identifying these priming effects and instantiating them in the priming model are worthy objectives for future research.

Target- and response-related effects. Our present formulation of the priming model focuses on cue-related effects, but it could be extended to account for target- and response-related effects. Target repetition effects could be modeled by assuming that target encoding is subject to priming in the same way as cue encoding. Residual activation of the target from the preceding trial could facilitate target encoding on the present trial if the target repeats. In the priming model, such priming of target encoding could be represented by changes in $R T_{\text {Base }}$, consistent with the modeling of hypothesized differences in target processing time in Model 3 of Logan and Bundesen (2004; also see Arrington \& Logan, 2004).

Response-related effects are more likely to affect compound cue retrieval or response execution than cue or target encoding processes. Many studies involving a 2:1 mapping of response categories onto response keys have generated response congruency effects: RT is faster when the correct response categorizations of a target for each task are mapped onto the same response key compared with different response keys (Meiran, 1996; Monsell et 
al., 2003). For example, there was a response congruency effect of $41 \mathrm{~ms}$ in our first experiment. We did not model the response congruency data because we averaged over a set of random walks for compound cue retrieval, but when RTs are calculated from separate random walks involving differential mappings of response categories onto response keys (resulting in different drift rates in Equation 5), the priming model fit to the first experiment produces a response congruency effect of about $20 \mathrm{~ms}$ - without changing any parameter values. The effect arises from a higher drift rate for compound cue retrieval on response congruent trials compared with response incongruent trials.

Many studies have also produced response repetition effects that interact with transition: There is a response repetition benefit for task repetitions and sometimes a response repetition cost for task alternations, although primarily in accuracy (e.g., Rogers \& Monsell, 1995). Across our three experiments, there was a response repetition benefit of $57 \mathrm{~ms}$ for cue repetitions, but response repetition costs of $-6 \mathrm{~ms}$ for task repetitions and $-26 \mathrm{~ms}$ for task alternations. These data suggest that response repetition effects are linked to whether the cue repeats or changes across trials, at least in the explicit task-cuing procedure. The assumption about temporary cue-response associations introduced in the priming model to account for the interaction between cue-target congruency and transition could possibly be extended to account for the interaction between response repetition and transition. We believe that these extensions for capturing target- and response-related effects are reasonable for future development of the priming model.

Residual switch costs. As noted earlier, our priming account predicts no differences between transitions at an infinite SOA because cue encoding will no longer contribute to RT. Such a prediction is not unique to our model (see Sohn \& Anderson, 2001, pp. 774-775), but the mere presence of a "residual switch cost" at a long SOA does not necessarily falsify the model. Stochastic fluctuations in cue encoding time from trial to trial could result in a small proportion of trials with long cue encoding times (i.e., the upper tail of the cumulative distribution of cue encoding times). Differences between transitions due to priming of cue encoding would be attenuated at long SOAs compared with short SOAs, but they need not be zero. In the present experiments, the mean difference between task alternations and task repetitions at the $800-\mathrm{ms}$ SOA was $58 \mathrm{~ms}$; the priming model predicted a mean difference of $46 \mathrm{~ms}$. Residual switch costs at long SOAs could also reflect other factors that are not instantiated in the present formulation of the priming model. For example, any factor that affects target encoding, response selection, or response execution would alter $R T_{\text {Base }}$ and could produce residual switch costs. Changes in $R T_{\text {Base }}$ might be justified by modeling those processes in greater detail.

Internally cued performance. It could be argued that our priming account is limited to task-switching situations involving the explicit task-cuing procedure. We believe that a version of it has the potential to be applied to procedures that do not involve explicit cues. The critical factor to be considered is how subjects can produce a cue to be used with the target for compound cue retrieval. We argue that procedures without explicit cues require the formation of internal (and possibly implicit) cues in STM, which may be verbal codes or related representations.

For example, consider the alternating runs procedure, in which subjects perform different tasks in a predictable cycle based on the spatial position of the target (Rogers \& Monsell, 1995). It is likely that subjects map the spatial positions onto internal cues in STM linked to the different tasks (e.g., stimuli appearing at the top require Task $\mathrm{A}$, whereas stimuli at the bottom require Task B). On each trial, the spatial position of the target activates the internal cue in STM, which serves as a mediator to retrieve responses from long-term memory.

This idea is based on Logan and Bundesen's (2004) hypothesis concerning the differences between arbitrary and meaningful explicit cues in their study. They argued that performance with arbitrary cues might require access to a mediator such as a task name to determine an appropriate response. Mediators would represent internal cues that are directly linked to arbitrary explicit cues, which could function much like spatial position cues in the alternating runs procedure. Differences among transitions could arise from differential priming of internal cues rather than an executive control process. Task repetitions would involve access to the same internal cue, whereas task alternations would involve access to a different internal cue that may have partially decayed in STM. Residual switch costs might emerge from less priming of the internal cue for the alternate task than the repeated task.

An extended version of our priming account could potentially explain internally cued performance, but this would depend on being able to identify the internal cues hypothesized to reside in STM. Evidence for internal cues may be difficult to obtain because their existence can only be inferred and not observed (unlike explicit cues), but there are experimental manipulations that may prove useful in identifying internal cues. For example, if internal cues are verbal representations of task names, then articulatory suppression could impair retrieval of internal cues when switching tasks. This idea is consistent with the finding that switch cost is increased under articulatory suppression, particularly when explicit cues are arbitrary or absent (Baddeley, Chincotta, \& Adlam, 2001; Emerson \& Miyake, 2003; Miyake et al., 2004; Saeki \& Saito, 2004). We are optimistic that future research will be able to shed light on the nature of internal cues in task switching.

\section{Executive Control and Task Sets}

The results from the present study indicate that task-switching performance can be interpreted and modeled with a set of basic psychological processes, but we are not arguing that executive control is absent from performance. Such an argument would require an explanation of how subjects are able to set themselves to do experiments similar to those we have reported. Subjects must be able to interpret the experimental instructions, identify the set of cues and targets, and learn the associations between response categories and response keys. Part of this process may involve raising the activation of relevant retrieval pathways or suppressing irrelevant pathways. Subjects will also need to block out certain aspects of their environment (e.g., unused response keys, extraneous visual stimuli in the testing room, etc.) and the multitude of experiment-irrelevant thoughts that could impair their performance.

Although we argue that an executive control process such as task-set reconfiguration is not necessary to explain differences among transitions, executive control may be necessary to form a general or multipurpose task set at the beginning of an experiment that prepares the cognitive system in a way that permits lower- 
level processes such as cue encoding to function in a more or less reflexive manner (cf. Monsell, 2003). This general task set could be construed as the set of control parameters (e.g., biases and priorities) determined by the "homunculus" in theories of executive control (Logan \& Gordon, 2001). The general task set may be flexible enough to handle simple tasks that are similar in structure. For example, although odd-even and high-low judgments are considered to be different tasks on the surface, they can be interpreted as the same task at a deeper level and can be performed with the same task set. They both involve encoding a cue and a target, retrieving a response category from long-term memory, and executing the selected response. Our priming model exploits this commonality with a general task set that is sufficient for performing both tasks. Regardless of whether the cues or tasks repeat or alternate across trials, the same task set can be used. It is reasonable to assume that executive control is required to adopt this general task set, but executive control is not required on a trial-by-trial basis to reconfigure task sets because there is only one task set. ${ }^{6}$

We began this article by noting the flexibility of the cognitive system, but flexibility is not synonymous with control. A welldesigned system can be flexible if it can handle variations in the environment without needing to be adjusted frequently. We suggest that the "clever homunculus" that is responsible for executive control (Logan \& Bundesen, 2003, 2004) may be clever enough to set the cognitive system in a way that allows subordinate processes to carry out the stimulus-driven operations that enable performance without frequent intervention from top-down control processes.

\section{Strategies for Theory Development}

Our demonstration that differences among transitions in taskswitching performance can be modeled without relying on executive control processes does not rule out such processes. Our priming account does not falsify task-set reconfiguration or taskset priming; in principle, many aspects of it are compatible with such accounts (e.g., compound cue retrieval could be integrated into any theory of task switching). What we offer with our priming account is an example of a strategy for theory development based on parsimony, sufficiency, and testability. Such a strategy has its advantages and disadvantages, but it can be fruitful in facilitating scientific progress.

Our priming account is parsimonious in that it invokes simple processes of encoding and retrieval to explain task-switching performance. Such processes have been investigated by psychologists for decades and are fundamental to many theories of cognition. These processes are clearly defined in our priming account, enabling us to instantiate them in a mathematical model. Executive control processes such as task-set reconfiguration are less transparent, despite their intuitive appeal as explanations of switch cost (Monsell, 2003). Task-set reconfiguration has been argued by at least one author to include

shifting attention between stimulus attributes or elements, or between conceptual criteria, retrieving goal states (what to do) and conditionaction rules (how to do it) into procedural working memory (or deleting them), enabling a different response set and adjusting response criteria. [Task-set reconfiguration] may well involve inhibition of elements of the prior task-set as well as activation of the required task-set. (Monsell, 2003, p. 135)
If some or all of these processes compose task-set reconfiguration, then how they interact to produce switch cost has to be specified in greater detail for us to have a comprehensive theory of task switching. Formal modeling is not a requirement for developing such a theory, but it helps to determine precisely how switch cost might be produced. An advantage of the present formulation of our priming account is that the source of switch cost is clear: differential priming of cue encoding. A disadvantage is that switch cost is influenced by many factors and is unlikely to arise from a single source; in this sense, our priming account is incomplete.

We believe there are elements of truth to the priming account that enabled us to model some aspects of explicitly cued performance, but it is clear that we have not captured all facets of task-switching performance. This incompleteness is not surprising because it was not our intention to develop a general theory of task switching. Our goal in this study was to provide an alternative account of a few critical effects (e.g., "switch costs" and their reduction with preparation time) to help stimulate a critical debate regarding the mechanisms involved in task switching. It seems that in many studies, there is an implicit, default assumption that any difference between task alternations and task repetitions reflects task-set reconfiguration; we challenge this assumption.

Even if our priming account is incorrect, what we have shown in the present study is that it provides a sufficient account of task-switching performance. Researchers will need to refine their theories to distinguish between sufficient accounts of data that differ in their underlying mechanisms. Hypotheses can be developed that lead to different predictions, and experiments can be conducted to test those predictions. An advantage of our priming account is that it is testable: Its assumptions and mechanisms are explicitly defined and can be evaluated. The priming model can be fit to data to determine whether it adequately captures various task-switching phenomena. A goal of our research approach is to see how far our priming model can be extended before it fails to account for critical aspects of task-switching performance.

The preceding discussion raises a general issue concerning theory development that goes beyond task switching. When, as researchers, we are attempting to explain what we perceive to be complex phenomena, it is tempting to posit complex processes that guide behavior. But as we have demonstrated with our priming account, complex phenomena need not arise from complex processes; interactions of simpler processes may be sufficient. We believe that a critical part of theory development involves determining whether behavior can be explained by simple processes and positing complex processes only when necessary, an idea that was central to the cognitive revolution in psychology in the middle

\footnotetext{
${ }^{6} \mathrm{We}$ are not suggesting that executive control is never involved on a trial-by-trial basis in all situations. When modeling performance in the psychological refractory period procedure, Logan and Gordon (2001) shifted parameter settings (i.e., changed the task set) between the first and the second stimulus to enforce serial processing. When modeling shifts of spatial attention in an attention cuing procedure, Logan (in press) found that a model with an attention switching process (Model 1 of Logan \& Bundesen, 2003) fit better than a model lacking such a process (Model 2). Our priming model is formally related to these "reconfiguration" models in that it is written in the language of Logan and Gordon's theory of executive control, but it does not require reconfiguration to account for "taskswitching" effects.
} 
of the 20th century. When it became clear that psychological phenomena such as language, problem solving, and concept learning could not be explained solely by the conditioning principles and stimulus-response associations of behaviorism, it was necessary to introduce mental representations and processes as explanatory constructs. Now this idea seems to be reversed in some cases (particularly in the task-switching domain), and the onus has been placed on researchers who wish to explain phenomena with basic psychological processes to demonstrate that more complex processes may not be involved.

\section{Conclusion}

Our short-term priming account of explicitly cued performance is able to explain task-switching data without invoking executive control mechanisms, which stands in stark contrast to the prevalent view that task switching involves task-set reconfiguration. Basic psychological processes were sufficient to produce several effects, including so-called switch costs and their reduction with preparation time. Executive control may play a role in setting the stage for general performance, but we argue that it does not need to be invoked as an explanatory construct on a trial-by-trial basis. The strength of this argument is based partly on the fact that our priming account can be formally modeled, providing a clear demonstration of its relationship to experimental data. In accordance with other authors (e.g., Anderson, Reder, \& Lebiere, 1996), we believe that an integrative research approach involving experimentation and modeling can be powerful in shedding light on cognitive phenomena. An integrative approach may even allow us to determine if and when task switching requires executive control.

\section{References}

Allport, A., Styles, E. A., \& Hsieh, S. (1994). Shifting intentional set: Exploring the dynamic control of tasks. In C. Umiltà \& M. Moscovitch (Eds.), Attention and performance (Vol. 15, pp. 421-452). Cambridge, MA: MIT Press.

Allport, A., \& Wylie, G. (1999). Task-switching: Positive and negative priming of task-set. In G. W. Humphreys, J. Duncan, \& A. Treisman (Eds.), Attention, space and action (pp. 273-296). Oxford, England: Oxford University Press.

Allport, A., \& Wylie, G. (2000). Task switching, stimulus-response bindings, and negative priming. In S. Monsell \& J. Driver (Eds.), Control of cognitive processes: Attention and performance (Vol. 18, pp. 35-70). Cambridge, MA: MIT Press.

Altmann, E. M. (2002). Functional decay of memory for tasks. Psychological Research, 66, 287-297.

Altmann, E. M. (2003). Task switching and the pied homunculus: Where are we being led? Trends in Cognitive Sciences, 7, 340-341.

Altmann, E. M. (2004). The preparation effect in task switching: Carryover of SOA. Memory \& Cognition, 32, 153-163.

Altmann, E. M., \& Gray, W. D. (2002). Forgetting to remember: The functional relationship of decay and interference. Psychological Science, $13,27-33$.

Anderson, J. R., \& Matessa, M. (1997). A production system theory of serial memory. Psychological Review, 104, 728-748.

Anderson, J. R., Reder, L. M., \& Lebiere, C. (1996). Working memory: Activation limitations on retrieval. Cognitive Psychology, 30, 221-256.

Arbuthnott, K. D., \& Woodward, T. S. (2002). The influence of cue-task association and location on switch cost and alternating-switch cost. Canadian Journal of Experimental Psychology, 56, 18-29.

Arrington, C. M., Altmann, E. M., \& Carr, T. H. (2003). Tasks of a feather flock together: Similarity effects in task switching. Memory \& Cognition, 31, 781-789.

Arrington, C. M., \& Logan, G. D. (2004). Episodic and semantic components of the compound-stimulus strategy in the explicit task-cuing procedure. Memory \& Cognition, 32, 965-978.

Baddeley, A., Chincotta, D., \& Adlam, A. (2001). Working memory and the control of action: Evidence from task switching. Journal of Experimental Psychology: General, 130, 641-657.

Brass, M., \& von Cramon, D. Y. (2004). Decomposing components of task preparation with functional magnetic resonance imaging. Journal of Cognitive Neuroscience, 16, 609-620.

Bundesen, C. (1990). A theory of visual attention. Psychological Review, 97, 523-547.

Busemeyer, J. R. (1982). Choice behavior in a sequential decision-making task. Organizational Behavior and Human Performance, 29, 175-207.

De Jong, R. (2000). An intention-activation account of residual switch costs. In S. Monsell \& J. Driver (Eds.), Control of cognitive processes: Attention and performance (Vol. 18, pp. 357-376). Cambridge, MA: MIT Press.

Dosher, B. A., \& Rosedale, G. (1989). Integrated retrieval cues as a mechanism for priming in retrieval from memory. Journal of Experimental Psychology: General, 118, 191-211.

Emerson, M. J., \& Miyake, A. (2003). The role of inner speech in task switching: A dual-task investigation. Journal of Memory and Language, $48,148-168$.

Gilbert, S. J., \& Shallice, T. (2002). Task switching: A PDP model. Cognitive Psychology, 44, 297-337.

Gillund, G., \& Shiffrin, R. M. (1984). A retrieval model for both recognition and recall. Psychological Review, 91, 1-67.

Goschke, T. (2000). Intentional reconfiguration and involuntary persistence in task set switching. In S. Monsell \& J. Driver (Eds.), Control of cognitive processes: Attention and performance (Vol. 18, pp. 331-355). Cambridge, MA: MIT Press.

Hintzman, D. L. (1986). "Schema abstraction" in a multiple-trace memory model. Psychological Review, 93, 411-428.

Hommel, B., \& Prinz, W. (Eds.). (1997). Theoretical issues in stimulusresponse compatibility. Amsterdam: North-Holland.

Jersild, A. T. (1927). Mental set and shift. Archives of Psychology, 14(Whole No. 89).

Koch, I. (2003). The role of external cues for endogenous advance reconfiguration in task switching. Psychonomic Bulletin \& Review, 10, 488-492.

Koch, I., \& Allport, A. (in press). Cue-based preparation and stimulusbased priming of tasks in task switching. Memory \& Cognition.

Koch, I., Prinz, W., \& Allport, A. (2005). Involuntary retrieval in alphabetarithmetic tasks: Task-mixing and task-switching costs. Psychological Research, 69, 252-261.

Kramer, A. F., Hahn, S., \& Gopher, D. (1999). Task coordination and aging: Explorations of executive control processes in the task switching paradigm. Acta Psychologica, 101, 339-378.

Logan, G. D. (1985). Executive control of thought and action. Acta Psychologica, 60, 193-210.

Logan, G. D. (1988). Toward an instance theory of automatization. Psychological Review, 95, 492-527.

Logan, G. D. (2002). An instance theory of attention and memory. Psychological Review, 109, 376-400.

Logan, G. D. (2003). Executive control of thought and action: In search of the wild homunculus. Current Directions in Psychological Science, 12, 45-48.

Logan, G. D. (2004). Cumulative progress in formal theories of attention. Annual Review of Psychology, 55, 207-234.

Logan, G. D. (in press). The time it takes to switch attention. Psychonomic Bulletin \& Review.

Logan, G. D., \& Bundesen, C. (2003). Clever homunculus: Is there an endogenous act of control in the explicit task-cuing procedure? Journal of Experimental Psychology: Human Perception and Performance, 29, 575599 . 
Logan, G. D., \& Bundesen, C. (2004). Very clever homunculus: Compound stimulus strategies for the explicit task-cuing procedure. Psychonomic Bulletin \& Review, 11, 832-840.

Logan, G. D., \& Gordon, R. D. (2001). Executive control of visual attention in dual-task situations. Psychological Review, 108, 393-434.

Luce, R. D. (1959). Individual choice behavior. New York: Wiley.

Luce, R. D. (1963). Detection and recognition. In R. D. Luce, R. R. Bush, \& E. Galanter (Eds.), Handbook of mathematical psychology (pp. 103189). New York: Wiley.

Mayr, U. (2001). Age differences in the selection of mental sets: The role of inhibition, stimulus ambiguity, and response-set overlap. Psychology and Aging, 16, 96-109.

Mayr, U., \& Kliegl, R. (2000). Task-set switching and long-term memory retrieval. Journal of Experimental Psychology: Learning, Memory, and Cognition, 26, 1124-1140.

Mayr, U., \& Kliegl, R. (2003). Differential effects of cue changes and task changes on task-set selection costs. Journal of Experimental Psychology: Learning, Memory, and Cognition, 29, 362-372.

Meiran, N. (1996). Reconfiguration of processing mode prior to task performance. Journal of Experimental Psychology: Learning, Memory, and Cognition, 22, 1423-1442.

Meiran, N. (2000a). Modeling cognitive control in task-switching. Psychological Research, 63, 234-249.

Meiran, N. (2000b). Reconfiguration of stimulus task sets and response task sets during task switching. In S. Monsell \& J. Driver (Eds.), Control of cognitive processes: Attention and performance (Vol. 18, pp. 377399). Cambridge, MA: MIT Press.

Meiran, N., Chorev, Z., \& Sapir, A. (2000). Component processes in task switching. Cognitive Psychology, 41, 211-253.

Meiran, N., Levine, J., Meiran, N., \& Henik, A. (2000). Task set switching in schizophrenia. Neuropsychology, 14, 471-482.

Miyake, A., Emerson, M. J., Padilla, F., \& Ahn, J. (2004). Inner speech as a retrieval aid for task goals: The effects of cue type and articulatory suppression in the random task cuing paradigm. Acta Psychologica, 115, 123-142.

Monsell, S. (1996). Control of mental processes. In V. Bruce (Ed.), Unsolved mysteries of the mind (pp. 93-148). Hove, England: Erlbaum.

Monsell, S. (2003). Task switching. Trends in Cognitive Sciences, 7, $134-140$.

Monsell, S., \& Driver, J. (2000). Banishing the control homunculus. In S. Monsell \& J. Driver (Eds.), Control of cognitive processes: Attention and performance (Vol. 18, pp. 3-32). Cambridge, MA: MIT Press.

Monsell, S., Sumner, P., \& Waters, H. (2003). Task-set reconfiguration with predictable and unpredictable task switches. Memory \& Cognition, 31, 327-342.

Nelson, D. L., McEvoy, C. L., \& Schreiber, T. A. (1999). The University of South Florida word association, rhyme, and fragment norms. Retrieved February 5, 2004, from http://w3.usf.edu/FreeAssociation/

Norman, D. A., \& Shallice, T. (1986). Attention to action: Willed and automatic control of behavior. In R. J. Davidson, G. E. Schwartz, \& D. Shapiro (Eds.), Consciousness and self-regulation (Vol. 4, pp. 1-18). New York: Plenum Press.

Nosofsky, R. M. (1986). Attention, similarity, and the identificationcategorization relationship. Journal of Experimental Psychology: General, 115, 39-57.

Nosofsky, R. M., \& Palmeri, T. J. (1997). An exemplar-based random walk model of speeded classification. Psychological Review, 104, 266-300.

Psychology Software Tools. (2002). E-Prime (Version 1.1) [Computer software]. Pittsburgh, PA.

Raaijmakers, J. G. W., \& Shiffrin, R. M. (1981). Search of associative memory. Psychological Review, 88, 93-134.
Ratcliff, R. (1978). A theory of memory retrieval. Psychological Review, 85, 59-108.

Ratcliff, R. (2001). Diffusion and random walk processes. In International encyclopedia of the social and behavioral sciences (Vol. 6, pp. 3668 3673). Oxford, England: Elsevier.

Ratcliff, R., \& McKoon, G. (1988). A retrieval theory of priming in memory. Psychological Review, 95, 385-408.

Rogers, R. D., \& Monsell, S. (1995). Costs of a predictable switch between simple cognitive tasks. Journal of Experimental Psychology: General, 124, 207-231

Rubinstein, J. S., Meyer, D. E., \& Evans, J. E. (2001). Executive control of cognitive processes in task switching. Journal of Experimental Psychology: Human Perception and Performance, 27, 763-797.

Ruthruff, E., Remington, R. W., \& Johnston, J. C. (2001). Switching between simple cognitive tasks: The interaction of top-down and bottom-up factors. Journal of Experimental Psychology: Human Perception and Performance, 27, 1404-1419.

Saeki, E., \& Saito, S. (2004). Effect of articulatory suppression on taskswitching performance: Implications for models of working memory. Memory, 12, 257-271.

Salthouse, T. A., Fristoe, N., McGuthry, K. E., \& Hambrick, D. Z. (1998) Relation of task switching to speed, age, and fluid intelligence. Psychology and Aging, 13, 445-461.

Schneider, D. W., \& Logan, G. D. (in press). Priming cue encoding by manipulating transition frequency in explicitly cued task switching. Psychonomic Bulletin \& Review.

Shaffer, L. H. (1965). Choice reaction with variable S-R mapping. Journal of Experimental Psychology, 70, 284-288.

Shepard, R. N. (1957). Stimulus and response generalization: A stochastic model relating generalization to distance in psychological space. Psychometrika, 22, 325-345.

Sohn, M.-H., \& Anderson, J. R. (2001). Task preparation and task repetition: Two-component model of task switching. Journal of Experimental Psychology: General, 130, 764-778.

Sohn, M.-H., \& Anderson, J. R. (2003). Stimulus-related priming during task switching. Memory \& Cognition, 31, 775-780.

Sohn, M.-H., Ursu, S., Anderson, J. R., Stenger, V. A., \& Carter, C. S. (2000). The role of prefrontal cortex and posterior parietal cortex in task switching. Proceedings of the National Academy of Sciences, USA, 97, 13448-13453.

Spector, A., \& Biederman, I. (1976). Mental set and mental shift revisited. American Journal of Psychology, 89, 669-679.

Stoet, G., \& Snyder, L. H. (2004). Single neurons in posterior parietal cortex of monkeys encode cognitive set. Neuron, 42, 1003-1012.

Sudevan, P., \& Taylor, D. A. (1987). The cuing and priming of cognitive operations. Journal of Experimental Psychology: Human Perception and Performance, 13, 89-103.

Townsend, J. T., \& Ashby, F. G. (1983). The stochastic modeling of elementary psychological processes. New York: Cambridge University Press.

Waszak, F., Hommel, B., \& Allport, A. (2003). Task-switching and longterm priming: Role of episodic stimulus-task bindings in task-shift costs. Cognitive Psychology, 46, 361-413.

Waszak, F., Hommel, B., \& Allport, A. (2004). Semantic generalization of stimulus-task bindings. Psychonomic Bulletin \& Review, 11, 1027-1033.

Woodward, T. S., Bub, D. N., \& Hunter, M. A. (2002). Task switching deficits associated with Parkinson's disease reflect depleted attentional resources. Neuropsychologia, 40, 1948-1955.

Wylie, G., \& Allport, A. (2000). Task switching and the measurement of "switch costs." Psychological Research, 63, 212-233.

Yeung, N., \& Monsell, S. (2003). Switching between tasks of unequal familiarity: The role of stimulus-attribute and response-set selection. Journal of Experimental Psychology: Human Perception and Performance, 29, 455-469. 


\section{Appendix A}

Mean Response Time (RT) and Accuracy (Percentage of Correct Responses) Across Subjects in Experiment 1

\begin{tabular}{|c|c|c|c|c|c|c|c|c|c|c|c|c|}
\hline \multirow[b]{3}{*}{ Transition } & \multirow{3}{*}{$\begin{array}{l}\text { Cue-target } \\
\text { congruency }\end{array}$} & \multirow[b]{3}{*}{ Measure } & \multicolumn{10}{|c|}{ Stimulus onset asynchrony (ms) } \\
\hline & & & \multicolumn{2}{|c|}{0} & \multicolumn{2}{|c|}{100} & \multicolumn{2}{|c|}{200} & \multicolumn{2}{|c|}{400} & \multicolumn{2}{|c|}{800} \\
\hline & & & $M$ & $S E$ & $M$ & $S E$ & $M$ & $S E$ & $M$ & $S E$ & $M$ & $S E$ \\
\hline \multirow[t]{4}{*}{ Cue repetition } & \multirow[t]{2}{*}{ Incongruent } & $\mathrm{RT}(\mathrm{ms})$ & 1,094 & 38 & 1,000 & 42 & 937 & 38 & 855 & 42 & 818 & 47 \\
\hline & & Accuracy & 96.8 & 0.9 & 96.8 & 0.8 & 98.1 & 0.5 & 97.0 & 0.7 & 95.7 & 1.3 \\
\hline & \multirow[t]{2}{*}{ Congruent } & RT (ms) & 1,030 & 42 & 939 & 43 & 898 & 42 & 781 & 36 & 791 & 40 \\
\hline & & Accuracy & 98.0 & 0.7 & 97.7 & 0.7 & 97.9 & 0.6 & 97.6 & 0.8 & 97.9 & 0.6 \\
\hline \multirow{4}{*}{ Task repetition } & \multirow{2}{*}{ Incongruent } & RT (ms) & 1,295 & 45 & 1,230 & 42 & 1,102 & 44 & 978 & 50 & 942 & 53 \\
\hline & & Accuracy & 94.3 & 1.4 & 93.4 & 1.7 & 94.4 & 1.3 & 94.2 & 1.2 & 97.4 & 0.7 \\
\hline & \multirow[t]{2}{*}{ Congruent } & RT (ms) & 1,132 & 39 & 1,027 & 45 & 932 & 42 & 893 & 49 & 833 & 44 \\
\hline & & Accuracy & 98.8 & 0.7 & 99.0 & 0.4 & 98.5 & 0.6 & 98.4 & 0.5 & 98.2 & 0.6 \\
\hline \multirow{4}{*}{ Task alternation } & \multirow{2}{*}{ Incongruent } & RT (ms) & 1,362 & 41 & 1,256 & 44 & 1,167 & 46 & 1,089 & 50 & 984 & 48 \\
\hline & & Accuracy & 94.9 & 1.1 & 94.1 & 0.9 & 93.9 & 1.0 & 94.3 & 1.0 & 96.8 & 0.6 \\
\hline & \multirow{2}{*}{ Congruent } & RT (ms) & 1,216 & 42 & 1,118 & 43 & 1,042 & 42 & 925 & 44 & 877 & 42 \\
\hline & & Accuracy & 97.4 & 0.7 & 96.8 & 0.7 & 96.9 & 0.8 & 97.0 & 0.7 & 96.5 & 0.7 \\
\hline
\end{tabular}

\section{Appendix B}

Mean Response Time (RT) and Accuracy (Percentage of Correct Responses) Across Subjects in Experiment 2

\begin{tabular}{|c|c|c|c|c|c|c|c|c|c|c|c|c|}
\hline \multirow[b]{3}{*}{ Transition } & \multirow{3}{*}{$\begin{array}{l}\text { Cue-target } \\
\text { congruency }\end{array}$} & \multirow[b]{3}{*}{ Measure } & \multicolumn{10}{|c|}{ Stimulus onset asynchrony (ms) } \\
\hline & & & \multicolumn{2}{|c|}{0} & \multicolumn{2}{|c|}{100} & \multicolumn{2}{|c|}{200} & \multicolumn{2}{|c|}{400} & \multicolumn{2}{|c|}{800} \\
\hline & & & $M$ & $S E$ & $M$ & $S E$ & $M$ & $S E$ & $M$ & $S E$ & $M$ & $S E$ \\
\hline \multirow[t]{6}{*}{ Cue repetition } & \multirow[t]{2}{*}{ Incongruent } & RT (ms) & 1002 & 41 & 1013 & 48 & 893 & 47 & 791 & 47 & 751 & 38 \\
\hline & & Accuracy & 95.3 & 1.3 & 97.1 & 1.2 & 96.1 & 1.1 & 96.8 & 1.2 & 98.4 & 0.7 \\
\hline & \multirow[t]{2}{*}{ Congruent } & $\mathrm{RT}(\mathrm{ms})$ & 962 & 45 & 893 & 41 & 801 & 39 & 757 & 40 & 675 & 36 \\
\hline & & Accuracy & 98.3 & 0.8 & 99.0 & 0.8 & 97.1 & 1.1 & 98.8 & 0.6 & 95.8 & 1.3 \\
\hline & \multirow[t]{2}{*}{ Dual } & $\mathrm{RT}(\mathrm{ms})$ & 994 & 39 & 884 & 33 & 823 & 44 & 806 & 40 & 755 & 37 \\
\hline & & Accuracy & 97.4 & 1.2 & 98.1 & 0.8 & 98.4 & 1.0 & 97.6 & 0.8 & 99.0 & 0.6 \\
\hline \multirow[t]{6}{*}{ Task repetition } & \multirow[t]{2}{*}{ Incongruent } & $\mathrm{RT}(\mathrm{ms})$ & 1191 & 48 & 1101 & 37 & 1023 & 52 & 887 & 41 & 808 & 46 \\
\hline & & Accuracy & 93.8 & 1.2 & 93.7 & 0.9 & 94.7 & 1.0 & 94.9 & 1.0 & 95.7 & 1.0 \\
\hline & \multirow[t]{2}{*}{ Congruent } & $\mathrm{RT}(\mathrm{ms})$ & 1038 & 44 & 905 & 39 & 857 & 43 & 782 & 42 & 734 & 33 \\
\hline & & Accuracy & 98.7 & 0.5 & 97.7 & 0.9 & 98.5 & 0.6 & 97.4 & 0.9 & 97.1 & 0.7 \\
\hline & \multirow[t]{2}{*}{ Dual } & $\mathrm{RT}(\mathrm{ms})$ & 1147 & 40 & 1050 & 42 & 958 & 33 & 859 & 40 & 799 & 35 \\
\hline & & Accuracy & 98.0 & 0.6 & 97.4 & 0.8 & 97.5 & 0.7 & 97.4 & 0.7 & 98.0 & 0.6 \\
\hline \multirow{6}{*}{ Task alternation } & \multirow{2}{*}{ Incongruent } & $\mathrm{RT}(\mathrm{ms})$ & 1276 & 42 & 1216 & 48 & 1104 & 45 & 983 & 45 & 906 & 45 \\
\hline & & Accuracy & 88.8 & 1.6 & 92.1 & 1.0 & 90.9 & 1.4 & 91.4 & 1.2 & 94.6 & 0.9 \\
\hline & \multirow[t]{2}{*}{ Congruent } & $\mathrm{RT}(\mathrm{ms})$ & 1124 & 42 & 1054 & 45 & 968 & 44 & 860 & 41 & 820 & 41 \\
\hline & & Accuracy & 95.7 & 0.6 & 96.1 & 0.6 & 94.3 & 1.1 & 95.0 & 0.8 & 95.2 & 0.8 \\
\hline & \multirow[t]{2}{*}{ Dual } & RT (ms) & 1176 & 39 & 1175 & 46 & 1063 & 43 & 960 & 47 & 875 & 39 \\
\hline & & Accuracy & 96.3 & 1.0 & 95.0 & 0.8 & 94.9 & 0.7 & 94.1 & 1.2 & 95.6 & 0.8 \\
\hline
\end{tabular}


Appendix C

Mean Response Time (RT) and Accuracy (Percentage of Correct Responses) Across Subjects in Experiment 3

\begin{tabular}{|c|c|c|c|c|c|c|c|c|c|c|c|c|c|}
\hline \multirow[b]{3}{*}{ Transition } & \multirow{3}{*}{$\begin{array}{l}\text { Cue-target } \\
\text { congruency }\end{array}$} & \multirow{3}{*}{$\begin{array}{c}\text { Spatial } \\
\text { compatibility }\end{array}$} & \multirow[b]{3}{*}{ Measure } & \multicolumn{10}{|c|}{ Stimulus onset asynchrony (ms) } \\
\hline & & & & \multicolumn{2}{|c|}{0} & \multicolumn{2}{|c|}{100} & \multicolumn{2}{|c|}{200} & \multicolumn{2}{|c|}{400} & \multicolumn{2}{|c|}{800} \\
\hline & & & & $M$ & $S E$ & $M$ & $S E$ & $M$ & $S E$ & $M$ & $S E$ & $M$ & $S E$ \\
\hline \multirow[t]{12}{*}{ Cue repetition } & \multirow[t]{4}{*}{ Incongruent } & \multirow[t]{2}{*}{ Incompatible } & $\mathrm{RT}(\mathrm{ms})$ & 985 & 35 & 936 & 39 & 862 & 51 & 783 & 39 & 685 & 35 \\
\hline & & & Accuracy & 96.2 & 1.6 & 97.5 & 1.2 & 96.5 & 1.6 & 95.3 & 2.2 & 95.8 & 1.6 \\
\hline & & \multirow[t]{2}{*}{ Compatible } & $\mathrm{RT}(\mathrm{ms})$ & 1011 & 42 & 936 & 47 & 863 & 33 & 775 & 33 & 651 & 38 \\
\hline & & & Accuracy & 95.1 & 1.9 & 96.3 & 1.6 & 93.1 & 2.4 & 99.2 & 0.6 & 97.0 & 1.9 \\
\hline & \multirow{4}{*}{ Congruent } & \multirow[t]{2}{*}{ Incompatible } & $\mathrm{RT}(\mathrm{ms})$ & 942 & 43 & 925 & 44 & 807 & 35 & 757 & 58 & 664 & 27 \\
\hline & & & Accuracy & 98.3 & 1.1 & 99.4 & 0.6 & 97.9 & 1.2 & 97.6 & 1.4 & 97.2 & 1.4 \\
\hline & & \multirow[t]{2}{*}{ Compatible } & $\mathrm{RT}(\mathrm{ms})$ & 872 & 35 & 846 & 34 & 839 & 51 & 730 & 34 & 646 & 33 \\
\hline & & & Accuracy & 99.5 & 0.5 & 97.6 & 1.7 & 97.8 & 1.4 & 96.9 & 1.6 & 100 & 0.0 \\
\hline & \multirow[t]{4}{*}{ Dual } & \multirow[t]{2}{*}{ Incompatible } & RT (ms) & 919 & 46 & 950 & 51 & 869 & 54 & 739 & 91 & 638 & 33 \\
\hline & & & Accuracy & 94.6 & 3.7 & 99.9 & 0.1 & 96.3 & 2.5 & 98.9 & 0.9 & 93.1 & 4.0 \\
\hline & & \multirow[t]{2}{*}{ Compatible } & $\mathrm{RT}(\mathrm{ms})$ & 974 & 52 & 849 & 36 & 763 & 50 & 771 & 37 & 629 & 40 \\
\hline & & & Accuracy & 99.0 & 0.9 & 95.6 & 3.6 & 96.6 & 1.7 & 98.2 & 1.8 & 100 & 0.0 \\
\hline \multirow{12}{*}{ Task repetition } & \multirow{4}{*}{ Incongruent } & \multirow{2}{*}{ Incompatible } & $\mathrm{RT}(\mathrm{ms})$ & 1218 & 35 & 1091 & 33 & 968 & 40 & 819 & 31 & 779 & 33 \\
\hline & & & Accuracy & 91.8 & 1.7 & 93.0 & 1.7 & 91.0 & 2.0 & 93.4 & 1.7 & 94.8 & 1.7 \\
\hline & & Compatible & $\mathrm{RT}(\mathrm{ms})$ & 1159 & 36 & 1102 & 38 & 944 & 33 & 848 & 32 & 804 & 28 \\
\hline & & & Accuracy & 90.9 & 1.9 & 95.7 & 1.1 & 92.2 & 1.7 & 96.0 & 1.2 & 95.3 & 1.3 \\
\hline & Congruent & Incompatible & $\mathrm{RT}(\mathrm{ms})$ & 988 & 35 & 919 & 40 & 855 & 32 & 778 & 37 & 740 & 34 \\
\hline & & & Accuracy & 98.1 & 0.8 & 99.5 & 0.4 & 97.9 & 0.9 & 98.0 & 1.2 & 98.2 & 0.9 \\
\hline & & Compatible & $\mathrm{RT}(\mathrm{ms})$ & 1000 & 32 & 928 & 37 & 803 & 28 & 726 & 28 & 694 & 22 \\
\hline & & & Accuracy & 98.7 & 0.9 & 98.8 & 0.6 & 99.2 & 0.6 & 98.3 & 0.7 & 97.6 & 1.0 \\
\hline & Dual & Incompatible & $\mathrm{RT}(\mathrm{ms})$ & 1100 & 37 & 1005 & 30 & 937 & 32 & 821 & 36 & 763 & 33 \\
\hline & & & Accuracy & 97.6 & 0.7 & 97.9 & 0.9 & 98.1 & 0.8 & 96.9 & 1.0 & 97.7 & 1.0 \\
\hline & & Compatible & $\mathrm{RT}(\mathrm{ms})$ & 1055 & 27 & 992 & 35 & 936 & 34 & 813 & 34 & 729 & 30 \\
\hline & & & Accuracy & 97.7 & 0.9 & 97.6 & 1.1 & 97.9 & 0.9 & 96.3 & 1.1 & 97.7 & 0.8 \\
\hline Task alternation & Incongruent & Incompatible & RT (ms) & 1279 & 38 & 1201 & 40 & 1079 & 41 & 925 & 37 & 840 & 36 \\
\hline & & & Accuracy & 90.4 & 2.0 & 93.3 & 1.4 & 91.4 & 1.5 & 92.7 & 1.5 & 94.5 & 1.6 \\
\hline & & Compatible & $\mathrm{RT}(\mathrm{ms})$ & 1275 & 35 & 1185 & 46 & 1070 & 41 & 904 & 37 & 842 & 41 \\
\hline & & & Accuracy & 91.9 & 1.7 & 90.8 & 1.6 & 92.4 & 1.3 & 93.7 & 1.3 & 93.6 & 1.3 \\
\hline & Congruent & Incompatible & $\mathrm{RT}(\mathrm{ms})$ & 1131 & 36 & 1015 & 40 & 938 & 38 & 830 & 41 & 763 & 42 \\
\hline & & & Accuracy & 97.4 & 0.7 & 97.0 & 0.8 & 96.6 & 0.9 & 97.1 & 0.8 & 97.9 & 0.7 \\
\hline & & Compatible & $\mathrm{RT}(\mathrm{ms})$ & 1032 & 36 & 984 & 32 & 882 & 31 & 808 & 39 & 728 & 33 \\
\hline & & & Accuracy & 97.0 & 0.7 & 95.8 & 1.2 & 97.8 & 1.0 & 96.3 & 1.0 & 98.3 & 0.6 \\
\hline & Dual & Incompatible & $\mathrm{RT}(\mathrm{ms})$ & 1235 & 43 & 1132 & 47 & 1011 & 35 & 944 & 39 & 808 & 36 \\
\hline & & & Accuracy & 97.3 & 0.9 & 95.5 & 1.0 & 95.1 & 1.0 & 97.3 & 0.9 & 96.4 & 1.0 \\
\hline & & Compatible & $\mathrm{RT}(\mathrm{ms})$ & 1133 & 40 & 1100 & 38 & 993 & 39 & 874 & 32 & 795 & 36 \\
\hline & & & Accuracy & 96.3 & 0.9 & 95.7 & 1.1 & 97.0 & 1.0 & 97.3 & 0.8 & 96.4 & 0.8 \\
\hline
\end{tabular}

Received June 8, 2004

Revision received March 9, 2005

Accepted March 24, 2005

\section{Online Preview of JEP: General Articles}

Are you an APA member or affiliate who subscribes to JEP: General? If so, you now have online access to the most recently accepted articles before they appear in print. Articles accepted and scheduled for publication are available on the journal's Web site at least 2 months prior to print publication. Access to this feature is available at no charge via

\section{http://www.apa.org/journals/xge.htm}

to APA members and affiliates who subscribe to JEP: General. 